\title{
Photon Upconversion in a Glowing Metal-Organic Framework
}

\author{
Indranil Roy, ${ }^{\dagger},+$ Subhadip Goswami, ${ }^{\dagger,+}$ Ryan M. Young,,,+++ Itai Schlesinger,,,++ \\ Mohammad Rasel Mian, ${ }^{\dagger}$ Alan E. Enciso, ${ }^{\dagger}$ Xuan Zhang, ${ }^{\dagger}$ Jessica E. Hornick,${ }^{\dagger}$ Omar K. Farha,${ }^{\dagger}$ \\ Michael R. Wasielewski, ${ }^{\dagger,}$ Joseph T. Hupp $^{\dagger}$ J. Fraser Stoddart $^{\dagger, \xi^{*}}$ \\ †Department of Chemistry, Institute for Sustainability and Energy at Northwestern, \\ Northwestern University, 2145 Sheridan Road, Evanston, Illinois 60208-3113, USA. \\ ${ }^{\S}$ School of Chemistry, University of New South Wales, Sydney, NSW 2052, Australia.
}

*E-mail: stoddart@ northwestern.edu

\section{SUPPLEMENTARY INFORMATION}

\section{Table of Contents}

Section A. Materials / General Methods / Instrumentation $\quad$ S2

$\begin{array}{lll}\text { Section B. Synthetic Protocols } & \text { S4 }\end{array}$

Section C. Crystallographic Characterization $\quad$ S6

$\begin{array}{llr}\text { Section D. Steady-State Spectroscopy } & \text { S8 }\end{array}$

$\begin{array}{lll}\text { Section E. Transient Absorption Spectroscopy } & \text { S10 }\end{array}$

$\begin{array}{lll}\text { Section F. Steady-State Microscopy } & \text { S24 }\end{array}$

$\begin{array}{lll}\text { Section G. Transient Absorption Microscopy } & \text { S25 }\end{array}$

$\begin{array}{llr}\text { Section H. } & \text { References } & \text { S28 }\end{array}$ 


\section{Section A. Materials / General Methods / Instrumentation}

All chemicals and reagents were purchased from commercial suppliers (Aldrich or Fisher) and used without further purification. Thin-layer chromatography (TLC) was performed on silica gel 60 F254 (E. Merck). Column chromatography was carried out on silica gel 60F (Merck 9385, 0.040-0.063 mm). UV/Vis Absorption spectra were recorded using a UV-3600 Shimadzu spectrophotometer. Steady-state emission spectra were acquired using a HORIBA Nanolog spectrofluorometer equipped with an integrating sphere for absolute photoluminescence quantum yield determination. ${ }^{1} \mathrm{H}$ Nuclear magnetic resonance $\left({ }^{1} \mathrm{H}\right.$ NMR) spectra were recorded on a Bruker Avance III spectrometer, with working frequencies of $500 \mathrm{MHz}$.

Quantum yield measurement: The samples were excited at $602 \mathrm{~nm}$ after $15 \mathrm{~min}$ of purging with Ar, and an in-built laser was used as an excitation source for all experiments. Absolute upconversion photoluminescence quantum yields $\left(\Phi_{\mathrm{UCF}}\right)$ were measured in $\mathrm{MeCN}$, as defined by the following equation.

$$
\Phi_{\mathrm{UCF}}=\frac{\text { number of upconverted photons }}{\text { number of low energy photons absorbed }}=\frac{q_{p_{-} e m}}{q_{p_{-} a b s}}
$$

where $q_{p-e m}$ is the upconverted emission photon flux (photons $\cdot \mathrm{S}^{-1}$ ) and $q_{p-a b s}$ is the photon flux absorbed by the sensitizer species (photons $\cdot \mathrm{S}^{-1}$ ). All upconversion quantum yields described in this work are based on the theoretical maximum of 50\%. The laser power was controlled by the software (FluorEssence ${ }^{\mathrm{TM}}$ ), and the laser power was measured with a Newport 843-R power meter.

Transient absorption spectroscopy: The setup for transient absorption measurements has been described elsewhere. ${ }^{1}$ Photoexcitation pulses at $414 \mathrm{~nm}$ were obtained through a beta barium borate (BBO) crystal doubling the fundamental, and the $575 \mathrm{~nm}$ pulses were generated with a laboratory-constructed optical parametric amplifier. The pulse power for photoexcitation was attenuated to $\sim 1 \mu \mathrm{J} /$ pulse, using neutral density filters. The pump polarization was randomized, employing a commercial depolarizer (DPU-25-A, Thorlabs, Inc.) in order to eliminate any orientational dynamics contributions from the experiment. All the spectra were collected on a 
commercial spectrometer (Ultrafast Systems, LLC Helios and EOS spectrometers, for fsTA and nsTA, respectively). All samples were stirred to avoid localized heating or degradation effects. Optical densities were maintained around 0.5 at the excitation wavelength for all samples.

Prior to kinetic analysis, the transient absorption data are background/scatter-subtracted and chirpcorrected. The visible and NIR spectroscopic data sets were spectroscopically merged (Surface Xplorer 4, Ultrafast Systems, LLC). Kinetic analyses were performed using home-written programs in MATLAB ${ }^{2}$ and were based on a global fit to selected single-wavelength kinetics. The time-resolution is given as $w=300 \mathrm{fs}$ (full width at half maximum, FWHM). The assumption of a uniform instrument response across the frequency domain and a fixed time-zero $\left(t_{0}\right)$ is implicit in the global analysis.

Transient absorption microscopy: Visible fsTA microscopy was performed using a commercial Yb:KGW, 12 W, 100 kHz repetition rate amplified laser system (Spirit-HE, Spectra-Physics). The $1040 \mathrm{~nm}$ output of the laser was split in two. The first portion was sent to a double-pass linear delay line (Newport, IMS600LM) and then focused on an $8 \mathrm{~mm}$ thick undoped YAG crystal for white-light continuum generation to be used as the probe beam. The probe beam was then recollimated, and the fundamental was filtered out using a $1040 \mathrm{~nm}$ notch filter. The second portion of the laser output was used to seed a non-collinear optical parametric amplifier (Sprit-NOPA-3HSHS, Light-Conversion, LLC) to generate a $542 \mathrm{~nm}$ pump. The pump was circularly polarized using an achromatic quarter waveplate and modulated using an electro-optic amplitude modulator (EOM, EO-AM-NR-C4, Thorlabs), which was phase synchronized to the laser output and operated at $25 \mathrm{kHz}$. The EOM was followed by a linear wire-grid polarizer to complete the modulation. The pump and probe beams were co-axially combined using a 50:50 BS and sent into an infinitycorrected, 20x objective lens to be focused on a single crystal. The transmitted beam leaving the crystal was recollimated using an identical 20× objective lens and sent to a 90(R):10(T) BS. The transmitted part was focused on a CMOS camera for imaging the sample while the reflected part entered a home-built spectrometer. The polarization of the pump and the probe beams were varied independently using two achromatic HWPs. The pump (probe) energy on the sample was set to 
$200 \mathrm{pJ} /$ pulse (50 pJ/pulse) using two neutral density (ND) filters. The pump (probe) focused spot size (FWHM) on the sample was $2.5 \mu \mathrm{m}(1.2 \mu \mathrm{m})$.

PXRD instrumentation: The powder X-Ray diffraction pattern of the MOF sample was measured on the IMSERC X-ray Facility at Northwestern University on a STOE-STADIMP powder diffractometer equipped with an asymmetrically curved Germanium monochromator $(\mathrm{CuK} \alpha 1$ radiation, $\lambda=1.54056 \AA$ ) and one-dimensional silicon strip detector (MYTHEN2 1K from DECTRIS). In this instrument, a Cu-X-ray tube was used at $40 \mathrm{kV}$ and $40 \mathrm{~mA}$. The MOF sample was drop cast on a metallic mask with a $3 \mathrm{~mm}$ diameter hole and secured between two Kapton tapes. During the measurement, the holder was spun, and the data were obtained in transmission mode.

\section{Section B. Synthetic Protocols}

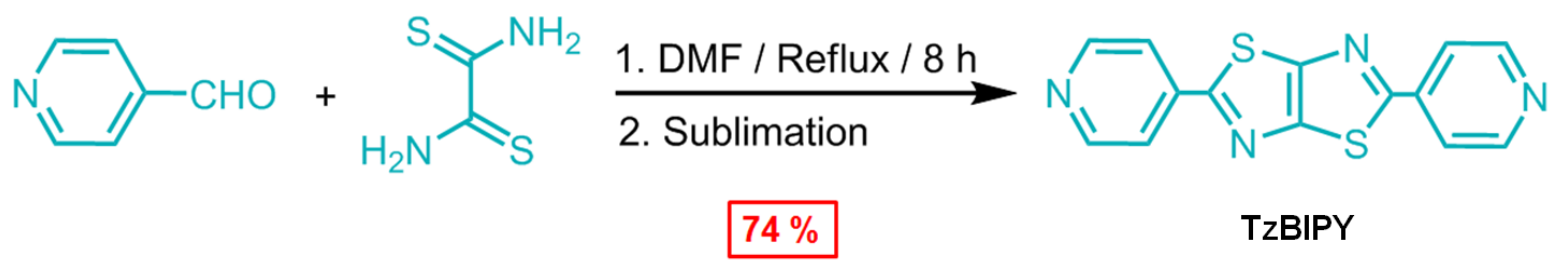

Scheme S1. Synthesis of 2,5-di(pyridin-4-yl)thiazolo[5,4-d]thiazole - TzBIPY

Synthesis of 2,5-Di(pyridin-4-yl)thiazolo[5,4-d]thiazole (TzBIPY): The synthetic protocol followed a literature procedure. ${ }^{3}$ A solution of dithiooxamide $(200 \mathrm{mg}, 1.6 \mathrm{mmol})$ and 4pyridinecarboxaldehyde $(0.40 \mathrm{~mL}, 4.4 \mathrm{mmol})$ in anhydrous dry DMF $(10 \mathrm{~mL})$ was heated under reflux for $4 \mathrm{~h}$ at $150^{\circ} \mathrm{C}$. Upon cooling, the product was precipitated out from the resulting solution. Filtration and washing with $\mathrm{H}_{2} \mathrm{O}$ afforded TzBIPY as yellow solid in $74 \%$ yield. ${ }^{1} \mathrm{H}$ NMR (500 $\left.\mathrm{MHz}, \mathrm{CDCl}_{3}, 25{ }^{\circ} \mathrm{C}\right) \delta=8.77(\mathrm{~d}, J=5.0 \mathrm{~Hz}, 2 \mathrm{H}), 7.86(\mathrm{~d}, J=6.0 \mathrm{~Hz}, 4 \mathrm{H}) \cdot{ }^{13} \mathrm{C} \mathrm{NMR}(125 \mathrm{MHz}$, $\left.\mathrm{CDCl}_{3}, 25^{\circ} \mathrm{C}\right) \delta=167.5,152.3,151.0,140.4,120.1$.

Synthesis of TzPMOF: In a 2-dram vial, TCPP $\left(5.1 \mathrm{mg}, 6.4 \times 10^{-3} \mathrm{mmol}\right)$ and $\mathrm{Zn}\left(\mathrm{NO}_{3}\right)_{2} .6 \mathrm{H}_{2} \mathrm{O}$ (5.8 $\mathrm{mg}, 0.03 \mathrm{mmol}$ ) were dissolved in DMF ( $1 \mathrm{~mL}$ ) by sonication (solution A). In another vial, 
hot DMF ( $3 \mathrm{~mL}$ ) was added to TzBIPY (7.4 $\mathrm{mg}, 0.024 \mathrm{mmol})$, and the hot solution passed through cotton balls to remove any undissolved residue (solution B). Solution A was then added to solution $\mathrm{B}$, and the reaction mixture was kept in an oven at $100^{\circ} \mathrm{C}$ for $24 \mathrm{~h}$. Dark red, rectangular plate-like crystals were observed on the walls and at the bottom of the vial after cooling the reaction mixture down to room temperature. After removing the solvent, fresh hot DMF was added to the MOF to remove any physisorbed linkers and kept for $2 \mathrm{~h}$. After repeating this step two more times, DMF was replaced by EtOH, and the sample was kept in EtOH until further use.

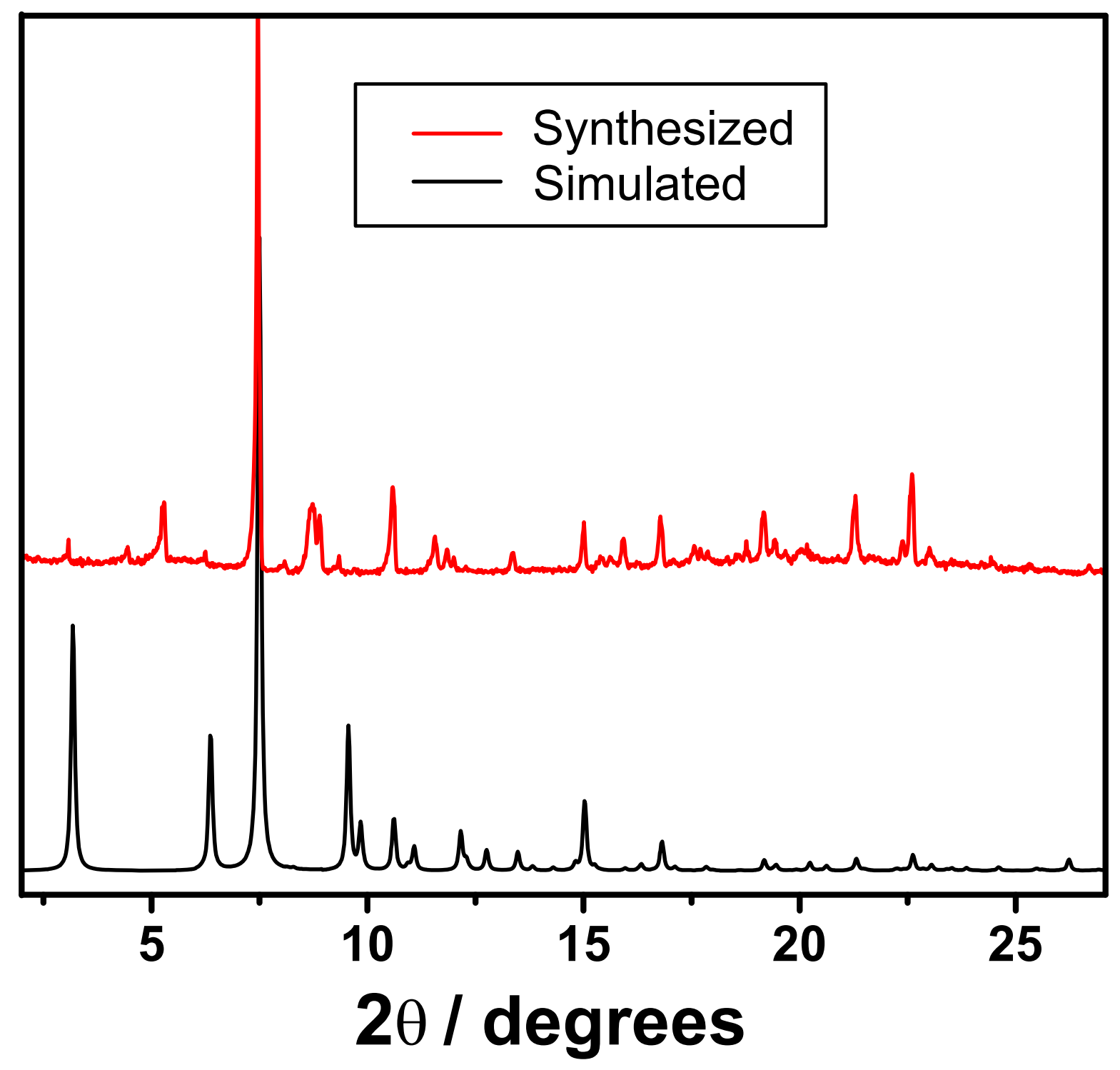

Figure S1. PXRD of TzPMOF 

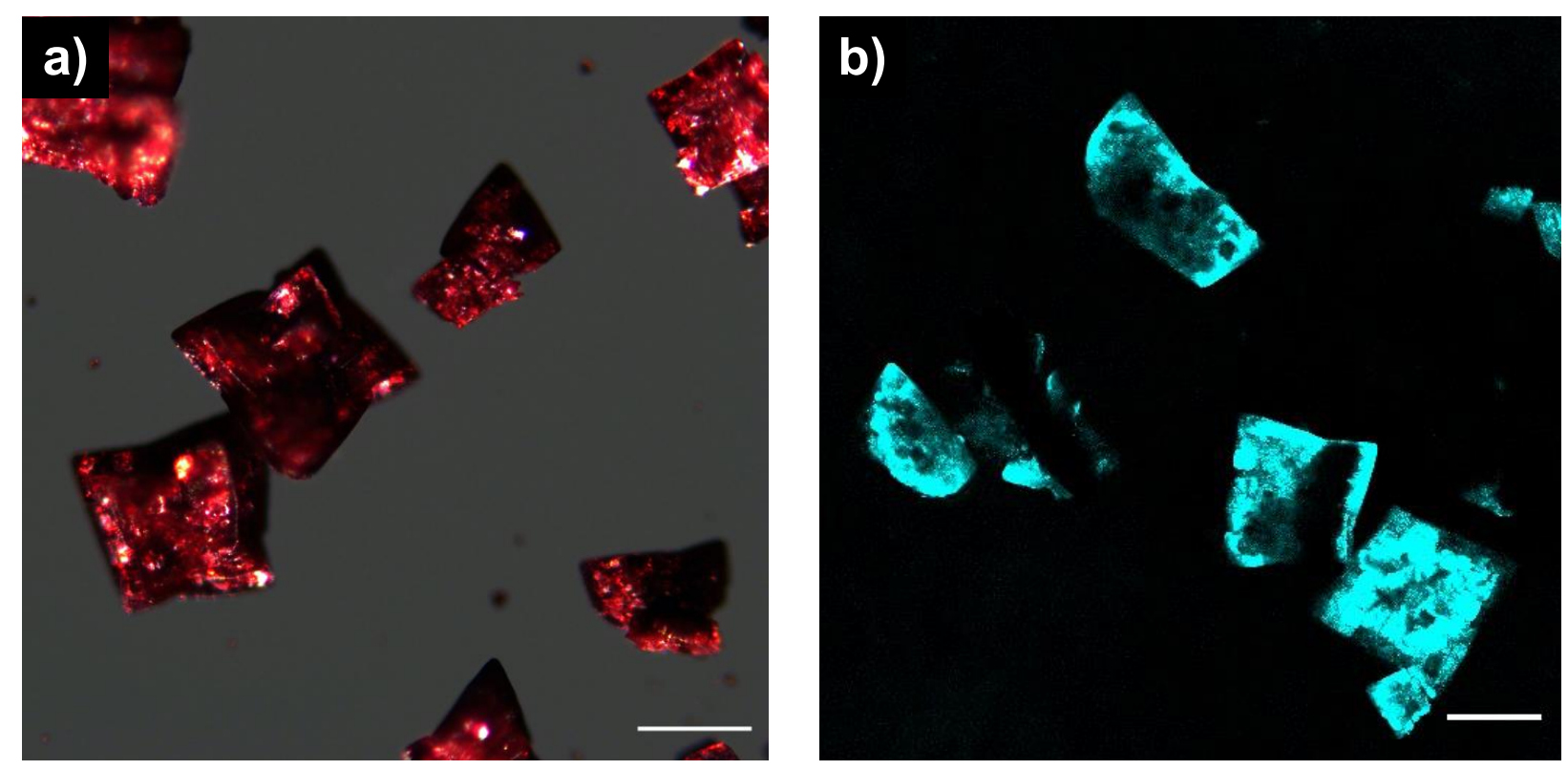

Figure S2. (a) Optical micrograph of the TzPMOF single-crystals. (b) Confocal micrograph of the TzPMOF single-crystals under excitation with $633 \mathrm{~nm}$ confocal lasers. The scale bar is 200 $\mu \mathrm{m}$. A lower upconversion fluorescence (UCF) intensity from TzPMOF single-crystals observed under the $633 \mathrm{~nm}$ excitation is caused by the inefficient excitation of the lowest energy Q band at $602 \mathrm{~nm}$.

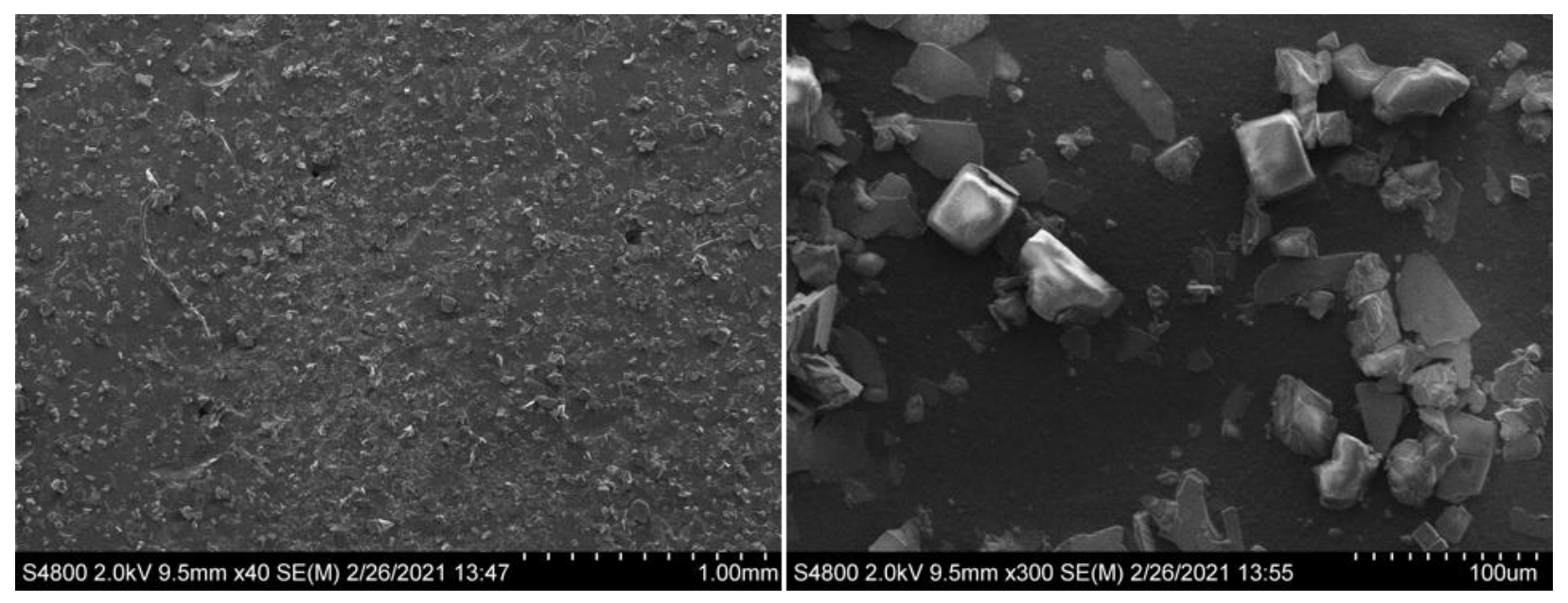

Figure S3. SEM micrographs of sonicated TzPMOF sample with average size $\sim 60 \mu \mathrm{M}$.

\section{Section C. Crystallographic Characterization}

Single-Crystal X-ray Structural Determination: The data were collected at $100 \mathrm{~K}$ on a 'Bruker APEX-II CCD' diffractometer with a $\mathrm{CuK} \alpha(\lambda=1.54178 \AA)$ microfocus X-ray source. The single crystal of TzPMOF was mounted on MicroMesh (MiTeGen) with paratone oil. The structures 
were determined by intrinsic phasing (SHELXT 2018/2) ${ }^{4}$ and refined by full-matrix least-squares refinement (SHELXL-2018/3) $)^{5}$ using the Olex ${ }_{2}^{6}$ software packages. The refinement results are summarized in Table S1. Crystallographic data for the TzPMOF in CIF format has been deposited in the Cambridge Crystallographic Data Centre (CCDC) under deposition numbers 2051613. The data can be obtained free of charge via www.ccdc.cam.ac.uk/data_request/cif (or from the Cambridge Crystallographic Data Centre, 12 Union Road, Cambridge CB2 1EZ, U.K.).

Table S1 Crystal data and structure refinement for TzPMOF.

\begin{tabular}{|c|c|}
\hline Empirical formula & $\mathrm{C}_{68} \mathrm{H}_{38} \mathrm{~N}_{8} \mathrm{O}_{9} \mathrm{~S}_{2} \mathrm{Zn}_{3}$ \\
\hline Formula weight & 1371.29 \\
\hline Temperature/K & 100.02 \\
\hline Crystal system & tetragonal \\
\hline Space group & $\mathrm{P} 4 / \mathrm{nmm}$ \\
\hline$a / \AA ̊$ & $16.6611(16)$ \\
\hline$b / \AA$ & $16.6611(16)$ \\
\hline$c / \AA ̊$ & $27.736(4)$ \\
\hline$\alpha /^{\circ}$ & 90 \\
\hline$\beta /^{\circ}$ & 90 \\
\hline$\gamma /{ }^{\circ}$ & 90 \\
\hline Volume $/ \AA^{3}$ & $7699.2(18)$ \\
\hline$Z$ & 2 \\
\hline$\rho_{\text {cald }} / \mathrm{gcm}^{-3}$ & 0.592 \\
\hline$\mu / \mathrm{mm}^{-1}$ & 1.015 \\
\hline $\mathrm{F}(000)$ & 1392.0 \\
\hline Crystal size $/ \mathrm{mm}^{3}$ & $0.08 \times 0.08 \times 0.02$ \\
\hline Radiation & $\mathrm{CuK} \alpha(\lambda=1.54178)$ \\
\hline $2 \Theta$ range for data collection ${ }^{\circ}$ & 6.188 to 86.028 \\
\hline Index ranges & $-7 \leq \mathrm{h} \leq 14,-11 \leq \mathrm{k} \leq 13,-24 \leq 1 \leq 23$ \\
\hline Reflections collected & 10243 \\
\hline Independent reflections & $1628\left[R_{\text {int }}=0.0530, R_{\text {sigma }}=0.0417\right]$ \\
\hline Data/restraints/parameters & $1628 / 414 / 167$ \\
\hline Goodness-of-fit on $\mathrm{F}^{2}$ & 1.469 \\
\hline Final $R$ indexes $[I>=2 \sigma(I)]$ & $R_{1}=0.1016, \mathrm{w} R_{2}=0.3157$ \\
\hline Final $R$ indexes [all data] & $R_{1}=0.1088, \mathrm{w} R_{2}=0.3252$ \\
\hline Largest diff. peak/hole / e $\AA^{-3}$ & $1.55 /-1.45$ \\
\hline
\end{tabular}




\section{Section D. Steady-State Spectroscopy}

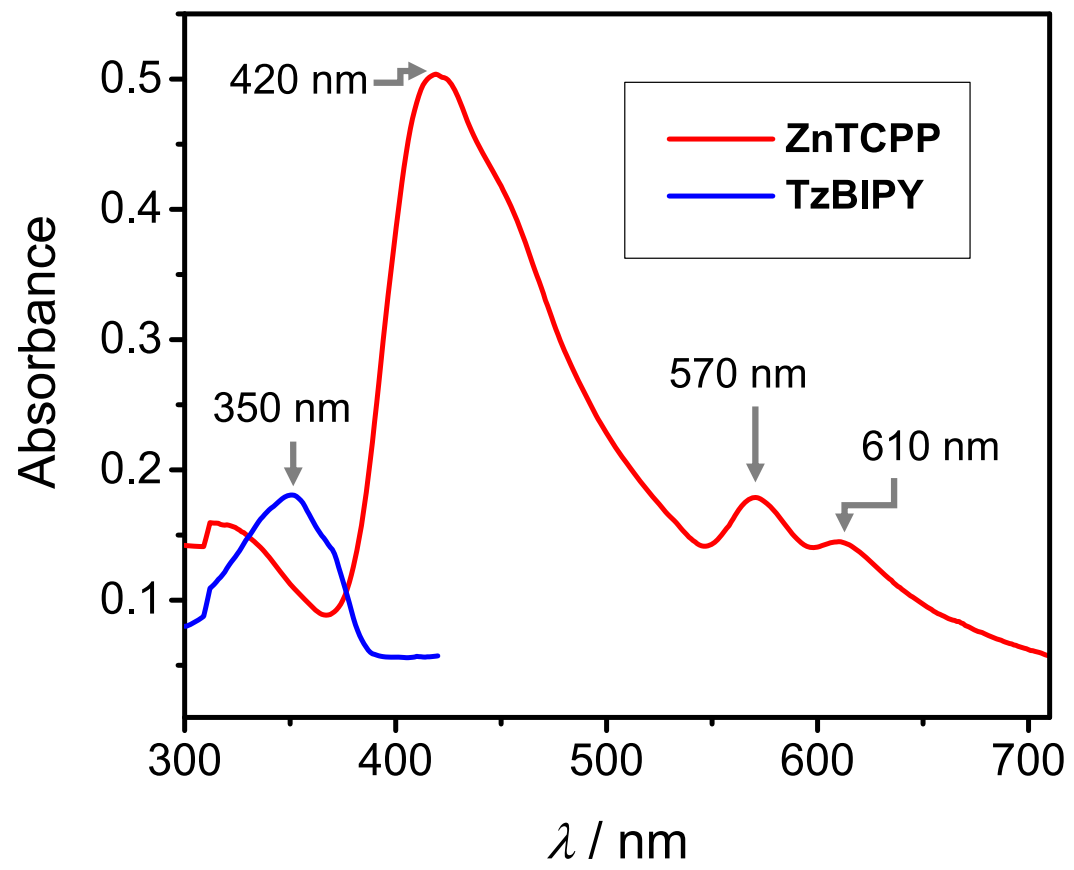

Figure S4. UV-Vis Spectra of TzBIPY and ZnTCPP in MeCN $(20 \mu \mathrm{M})$

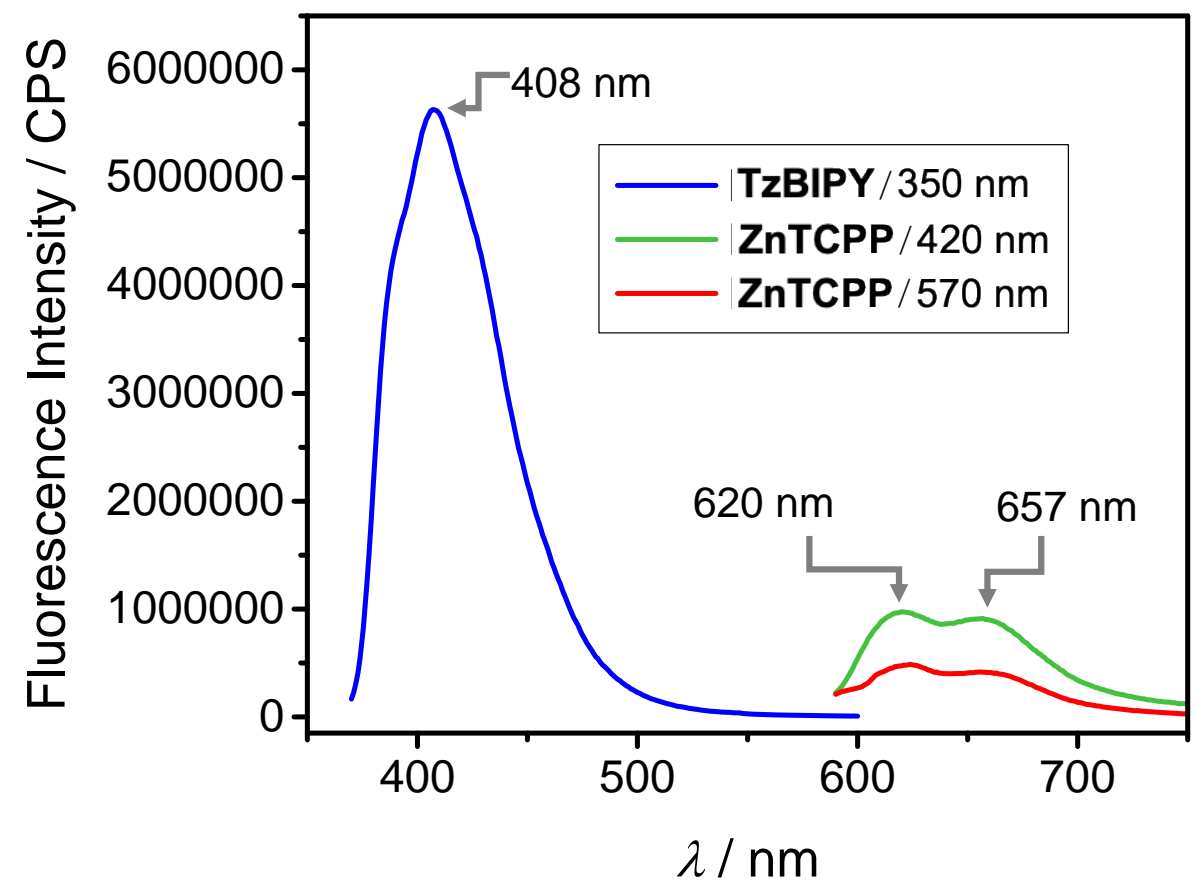

Figure S5. Emission spectra of TzBIPY and ZnTCPP in MeCN $(20 \mu \mathrm{M})$ 


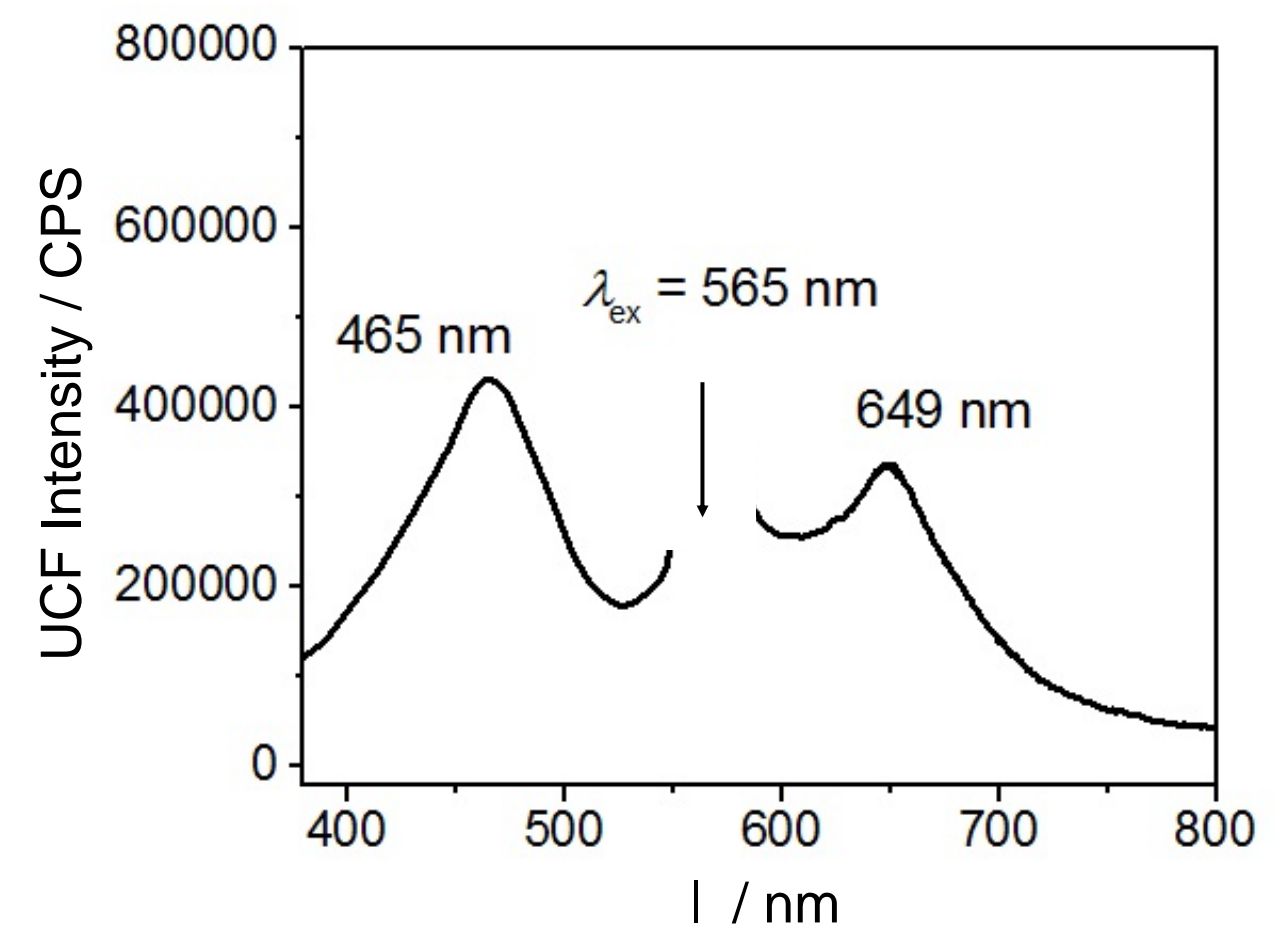

Figure S6. Emission spectra of TzPMOF in $\mathrm{Me}_{2} \mathrm{CO}$ suspension $(\lambda=565 \mathrm{~nm})$

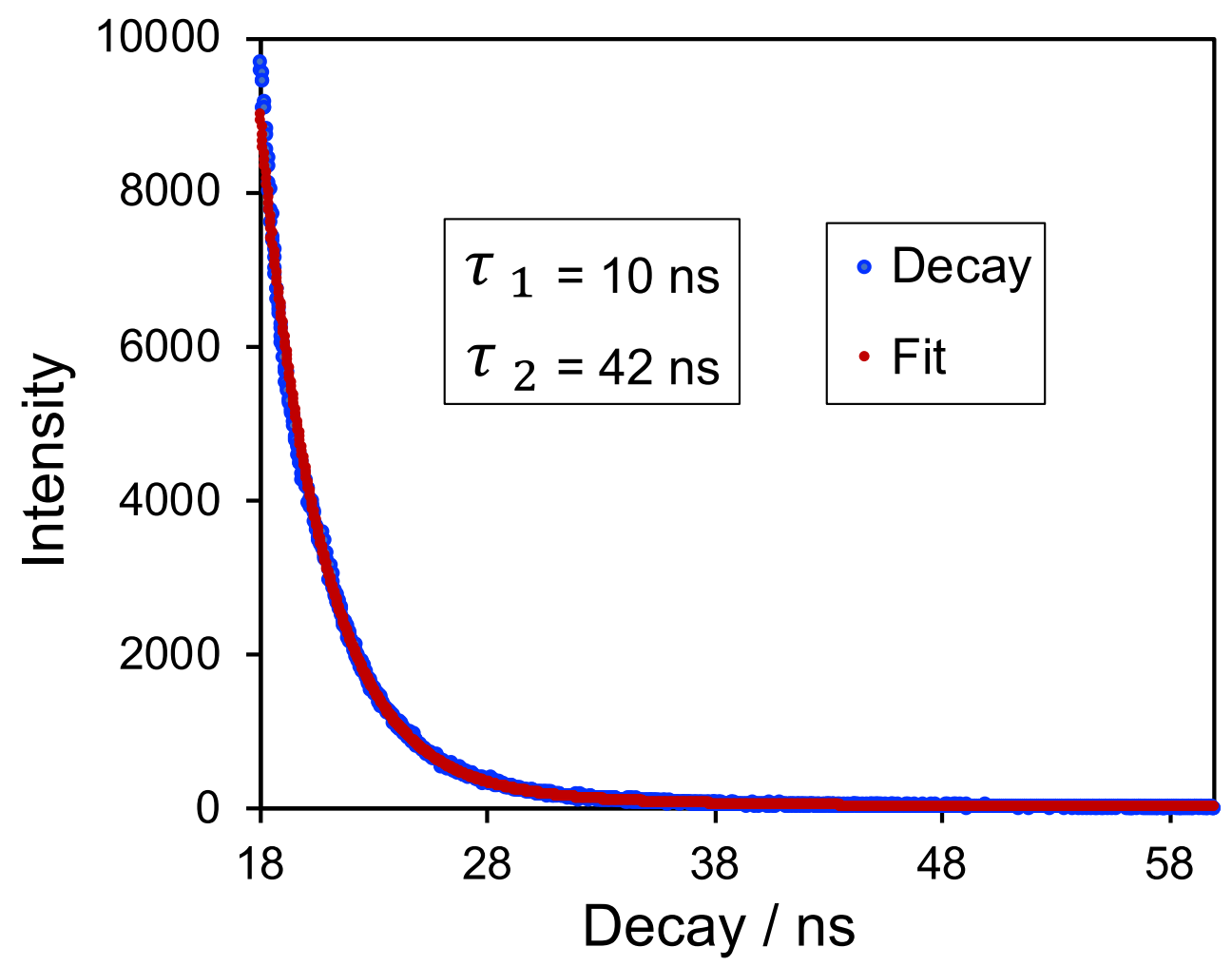

Figure S7. Fluorescence decay profile of TzPMOF in $\mathrm{Me}_{2} \mathrm{CO}$ suspension $\left(\lambda_{\mathrm{ex}}=565 \mathrm{~nm}\right)$ 
Section E. Transient Absorption Spectroscopy

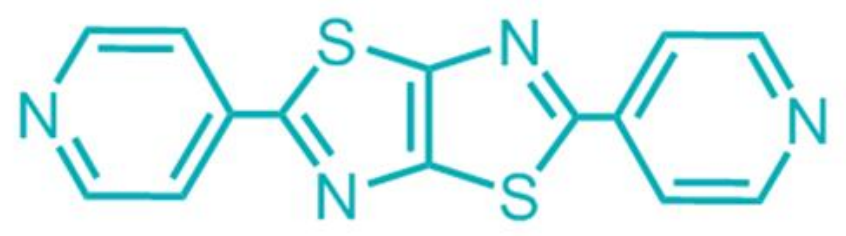

\section{TzBIPY}

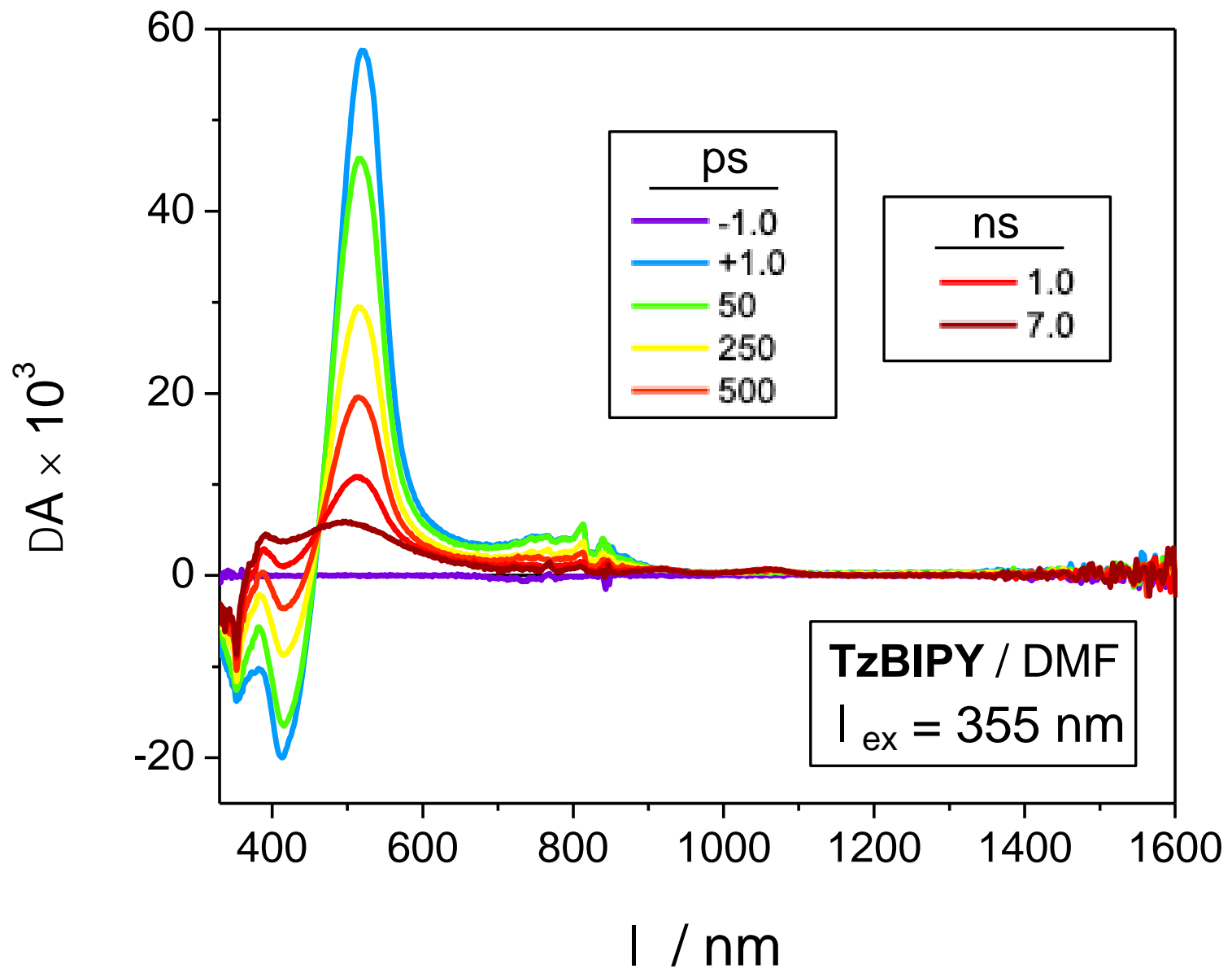

Figure S8. Femtosecond transient absorption spectra of TzBIPY in DMF excited at $\lambda_{\mathrm{ex}}=355 \mathrm{~nm}$ 
a)

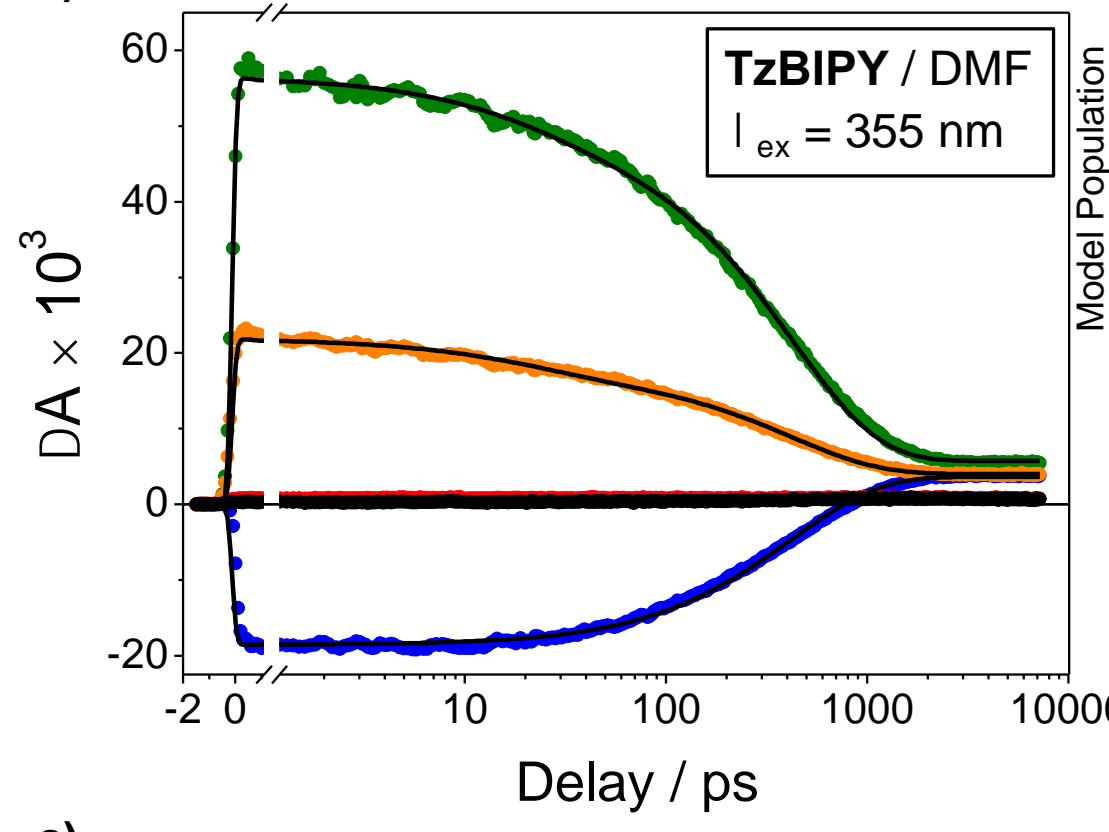

c)

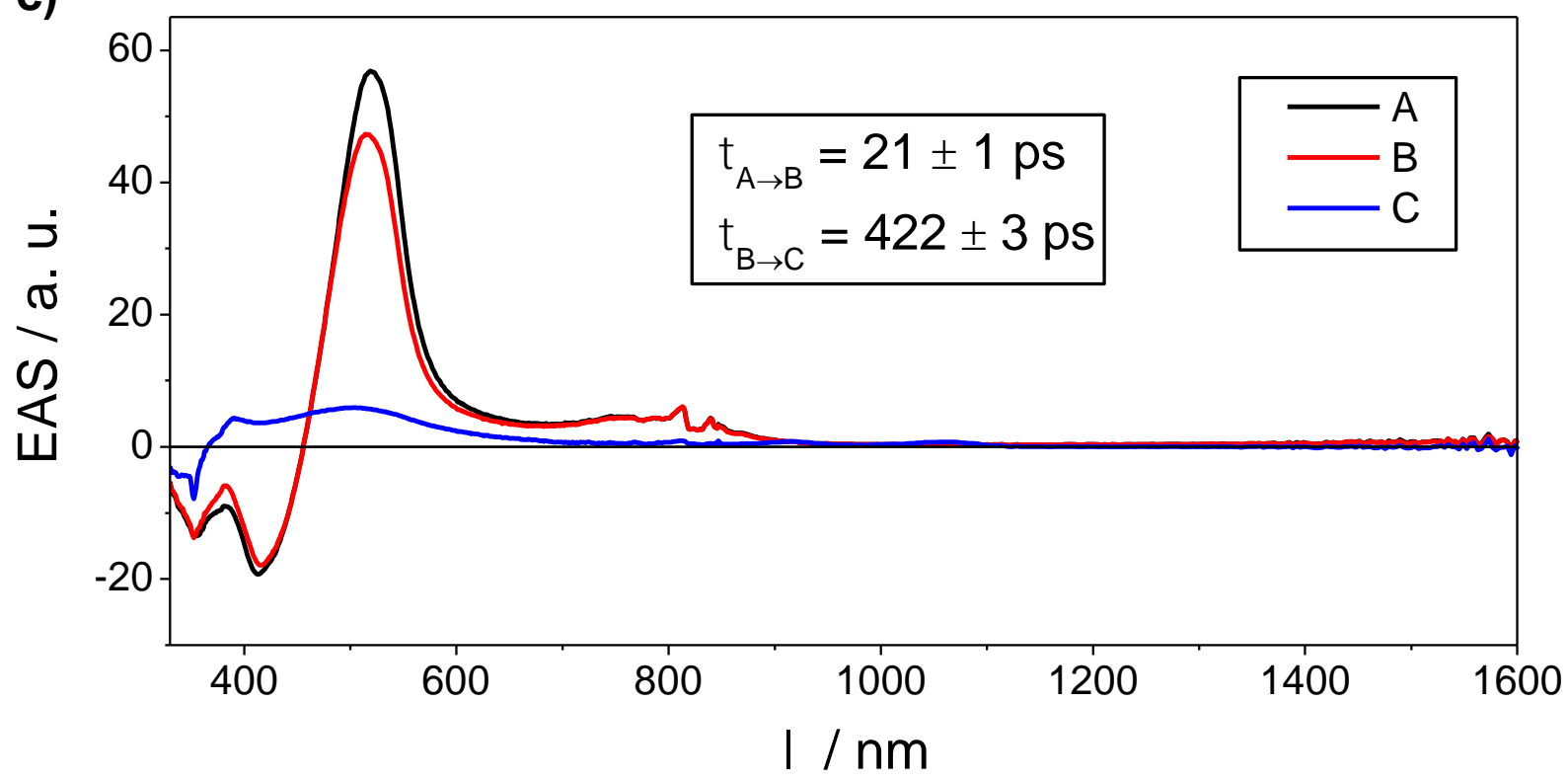

b)
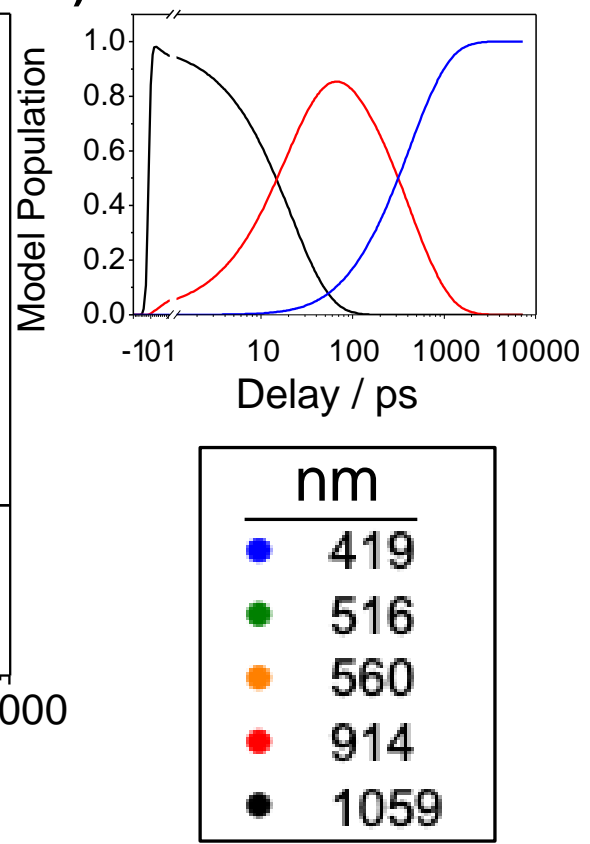

Figure S9. Kinetic analysis of the femtosecond transient data for TzBIPY in DMF excited at $\lambda_{\text {ex }}$ $=355 \mathrm{~nm}$. (a) Kinetic traces at selected wavelengths with fits (black lines). (b) Model populations for the fit to a three-state sequential kinetic model. (c) Evolution-associated spectra (EAS) for the three states in the model 
TA shows (Figure S9) the singlet state of the TzBIPY (A) first relaxes in $~ 21$ ps. Then this relaxed state (B) intersystem crosses in $\sim 0.4 \mathrm{~ns}$ to form the triplet state (C) that lives into the nsTA regime.

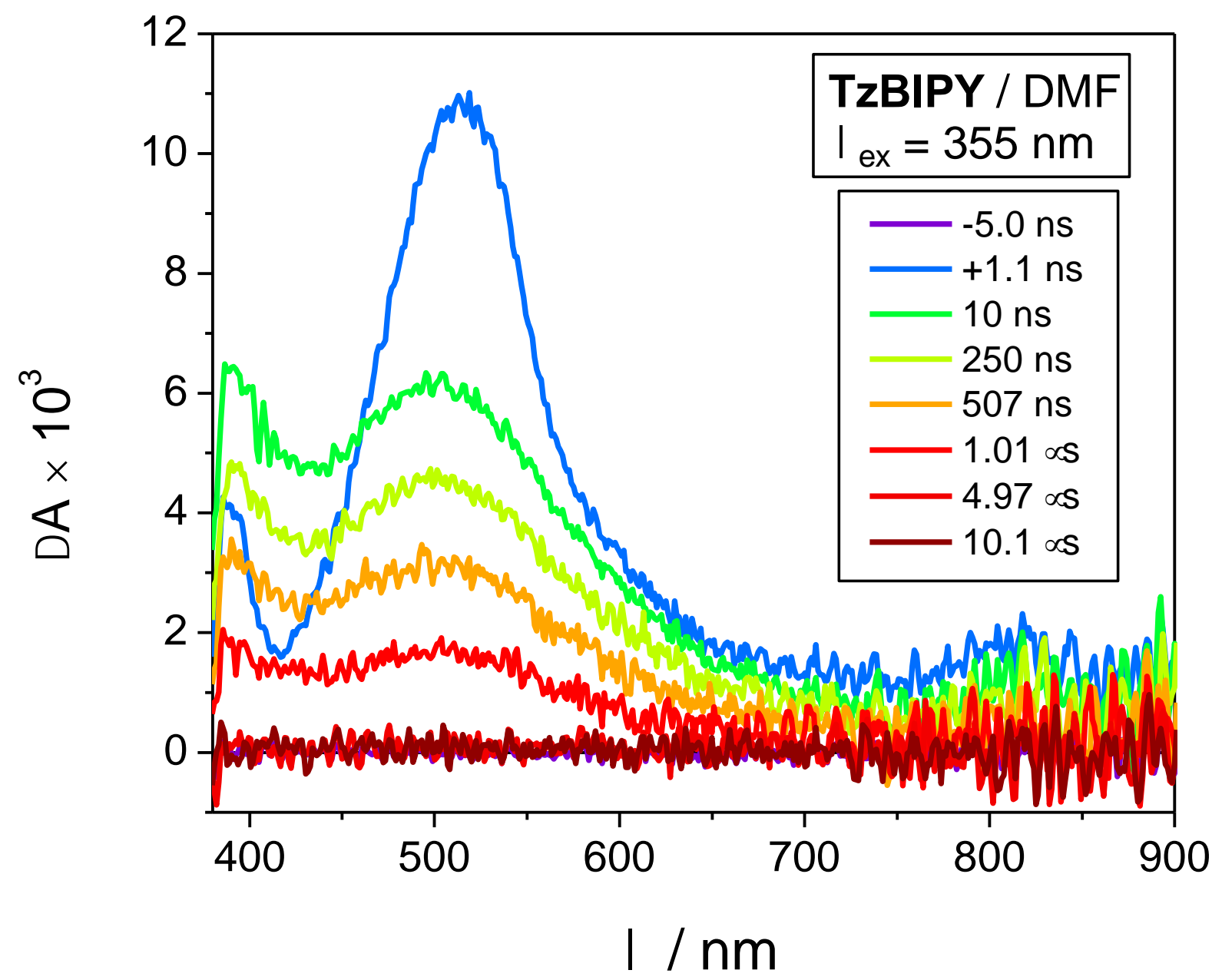

Figure S10. Nanosecond transient absorption spectra of TzBIPY in DMF excited at $\lambda_{\mathrm{ex}}=355 \mathrm{~nm}$

TA shows (Figure S11) the singlet state of the TzBIPY (B) intersystem crosses in $\sim 0.7 \mathrm{~ns}$ to form the triplet state (C) that decays to the ground state in $790 \mathrm{~ns}$. 
a)

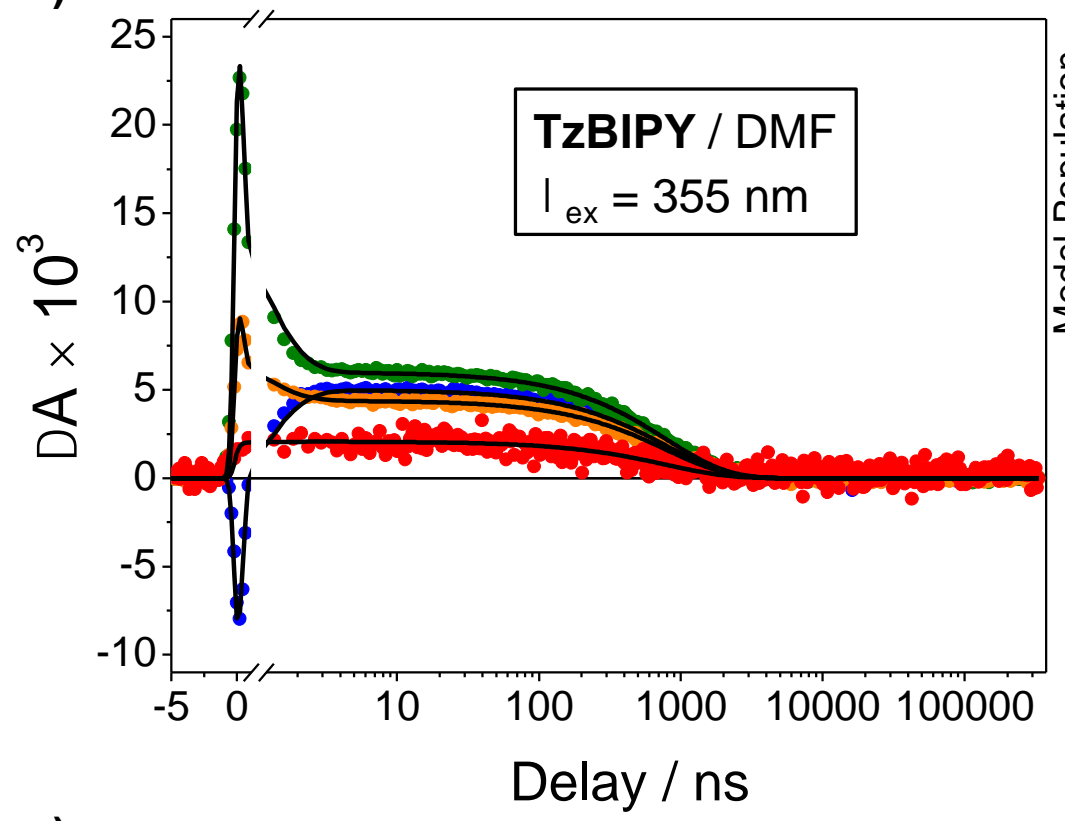

b)
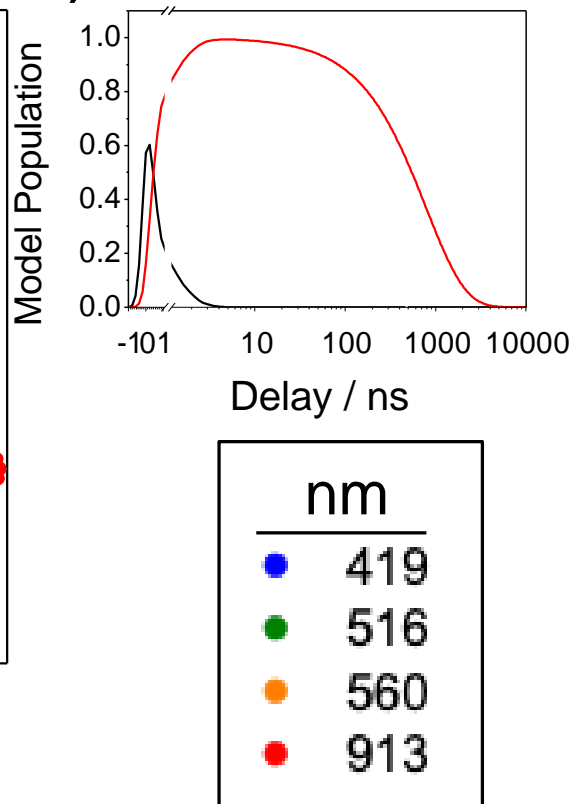

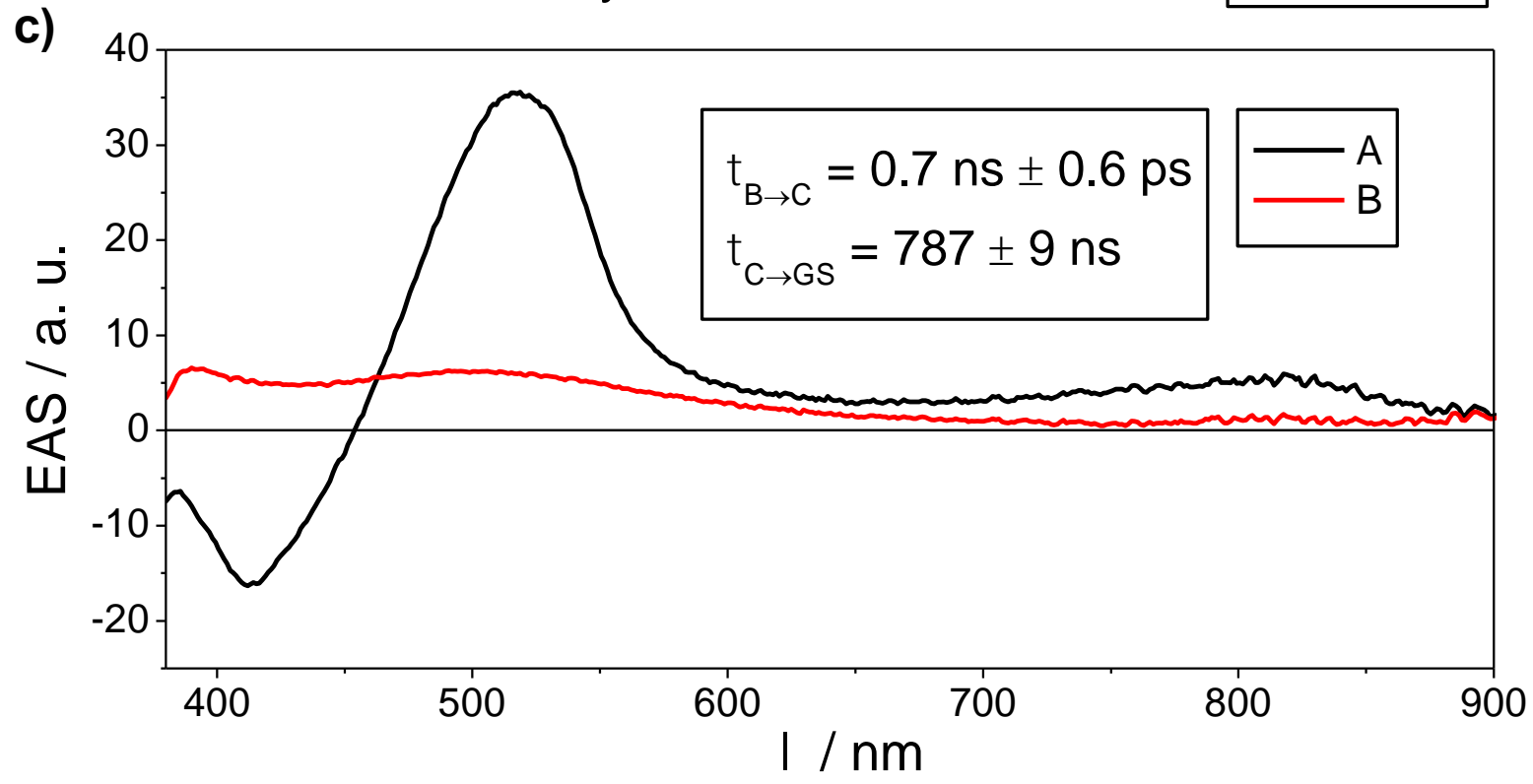

Figure S11. Kinetic analysis of nanosecond transient data for TzBIPY in DMF excited at $\lambda_{\mathrm{ex}}=$ $355 \mathrm{~nm}$. (a) Kinetic traces at selected wavelengths with fits (black lines). (b) Model populations for the fit to a two-state sequential kinetic model. (c) Evolution-associated spectra (EAS) for the three states in the model 

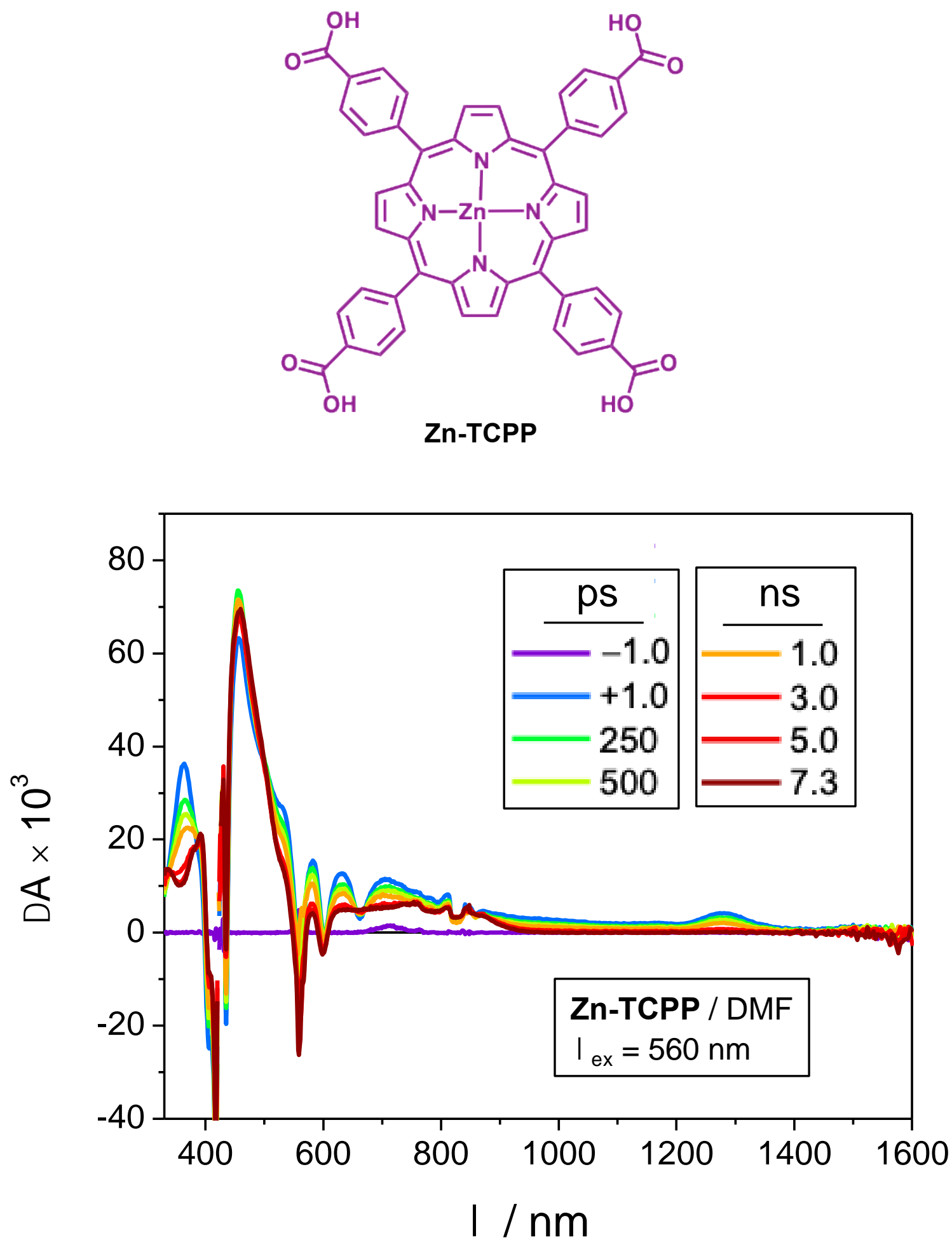

Figure S12. Femtosecond transient absorption spectra of Zn-TCPP in DMF excited at $\lambda_{\mathrm{ex}}=560 \mathrm{~nm}$ 
a)

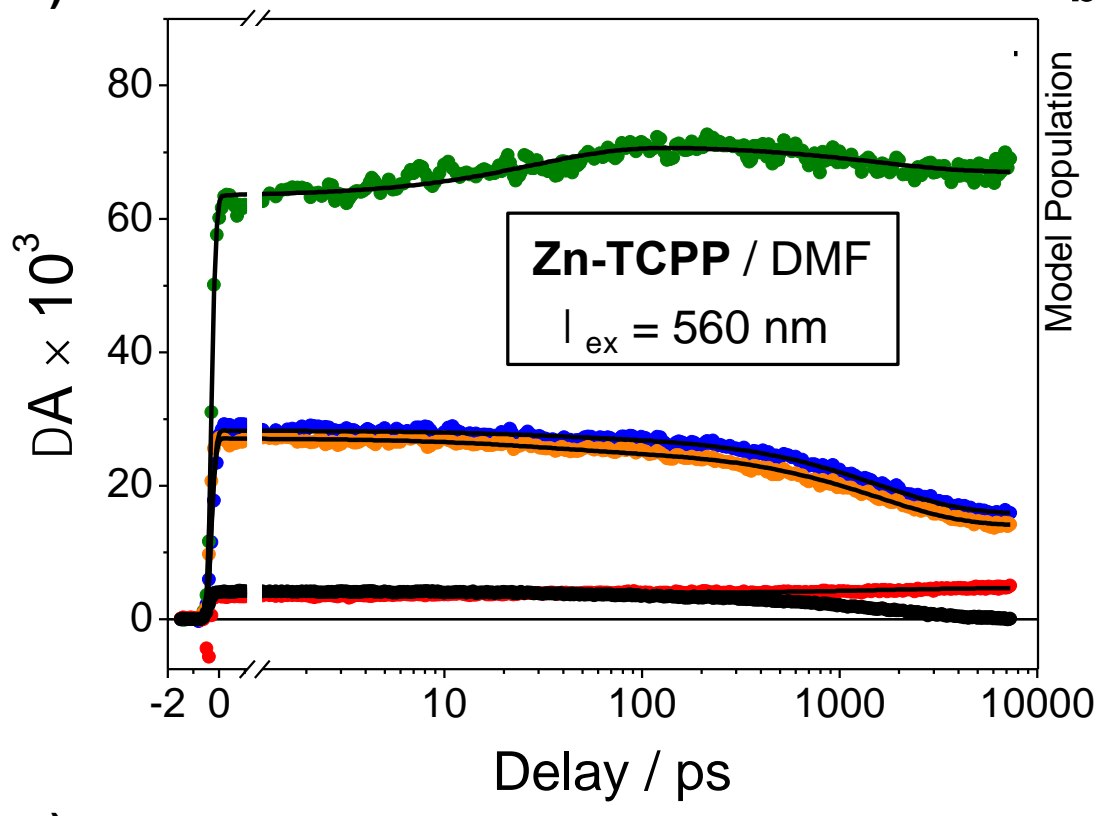

c)

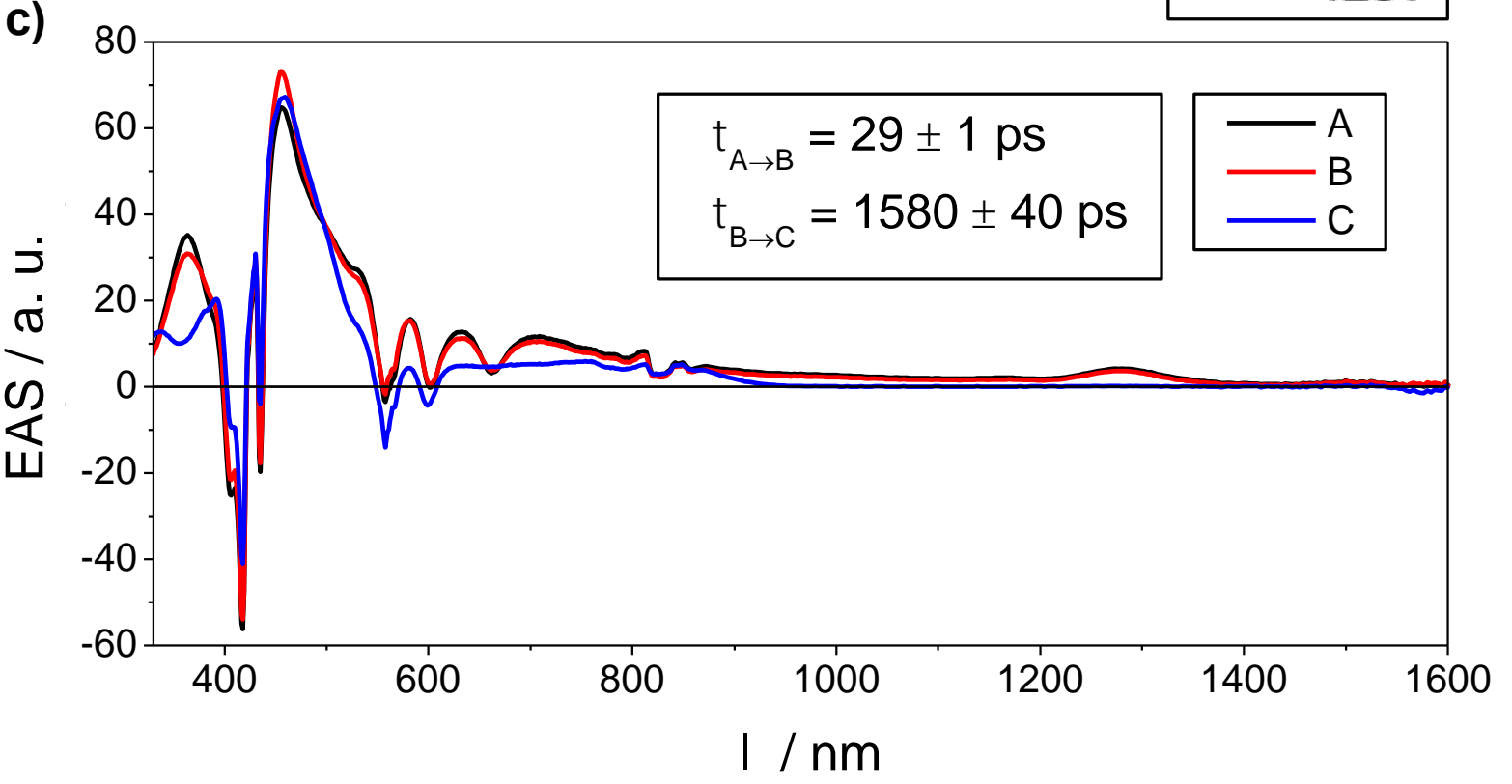

Figure S13. Kinetic analysis of femtosecond transient data for Zn-TCPP in DMF excited at $\lambda_{\mathrm{ex}}=$ $355 \mathrm{~nm}$. (a) Kinetic traces at selected wavelengths with fits (black lines). (b) Model populations for the fit to a three-state sequential kinetic model. (c) Evolution-associated spectra (EAS) for the three states in the model 
TA shows (Figure S13) the singlet state of $\mathbf{Z n - T C P P ~ ( A ) ~ i n ~ t h e ~ f i r s t ~ i n s t a n c e ~ r e l a x e s ~ i n ~} \sim 30 \mathrm{ps,}$ when the Q-band is excited. Then this relaxed state (B) intersystem crosses in $\sim 1.6 \mathrm{~ns}$ to form the triplet state $(\mathrm{C})$ that lives into the nsTA regime.

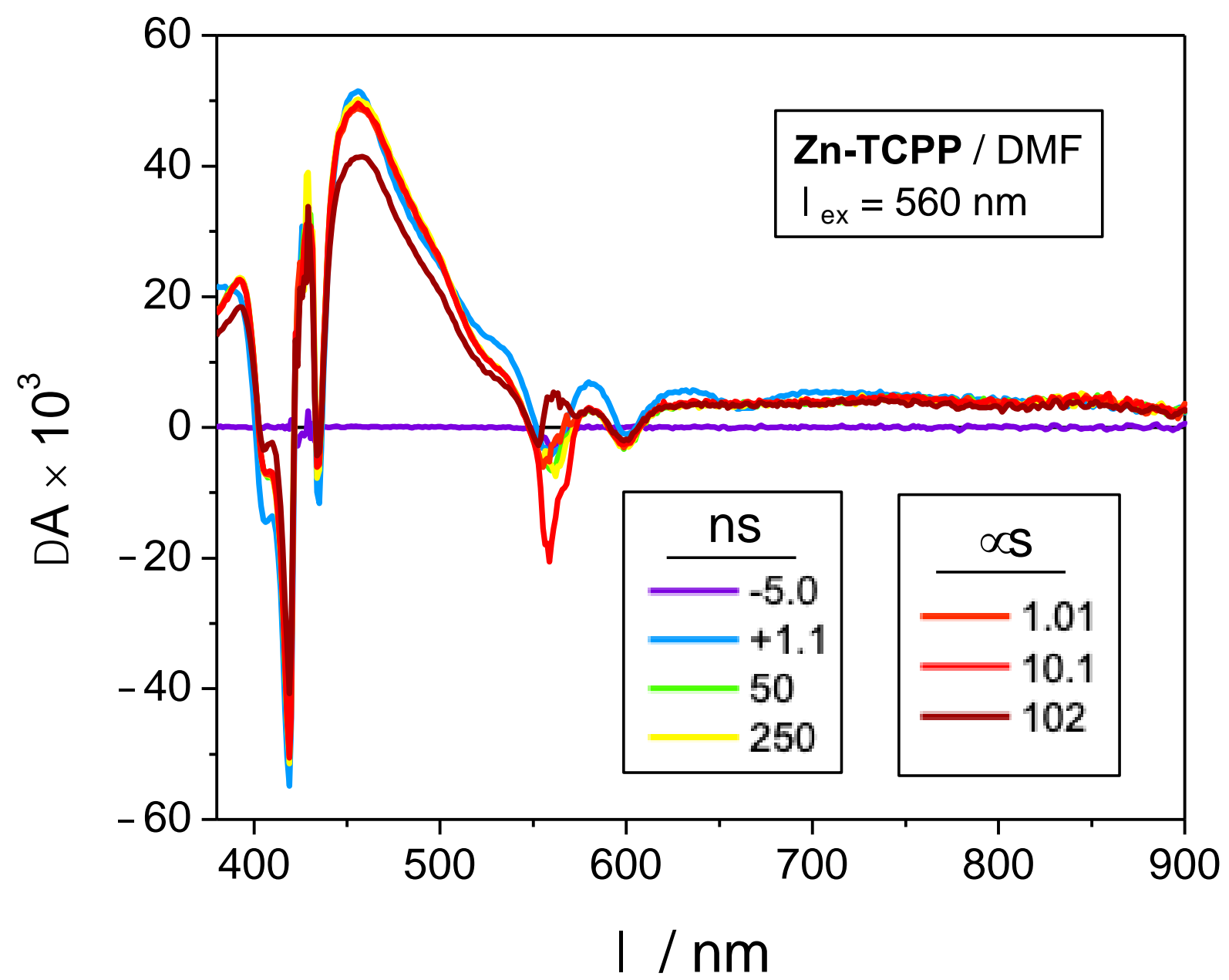

Figure S14. Nanosecond transient absorption spectra of Zn-TCPP in DMF excited at $\lambda_{\mathrm{ex}}=560 \mathrm{~nm}$

TA shows (Figure S15) the singlet state (C) intersystem crosses in $~ 2.6 \mathrm{~ns}$ to form the triplet state (D) that decays to the ground state in $670 \mu \mathrm{s}$. 


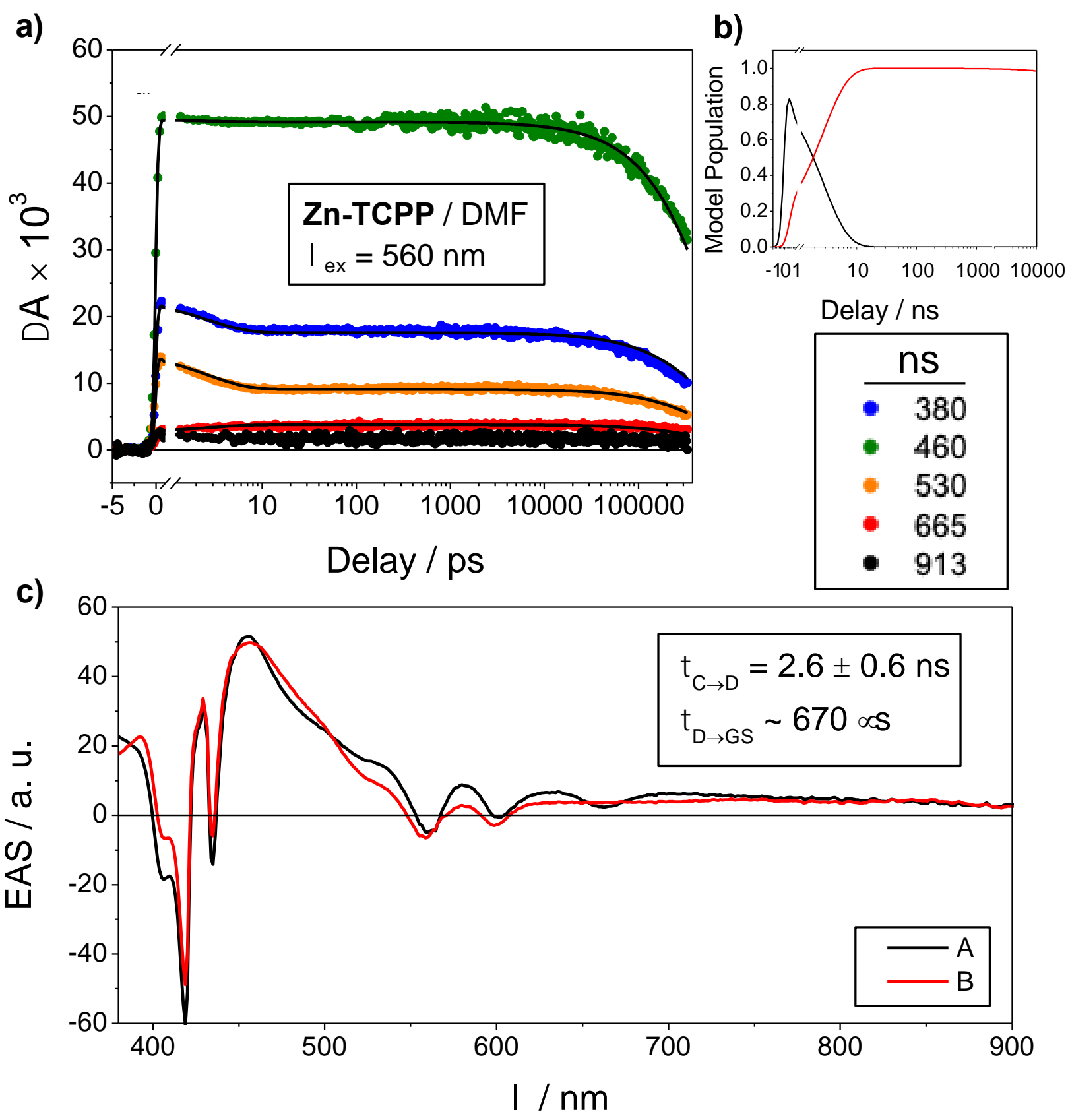

Figure S15. Kinetic analysis of nanosecond transient data for Zn-TCPP in DMF excited at $\lambda_{\mathrm{ex}}=$ $560 \mathrm{~nm}$. (a) Kinetic traces at selected wavelengths with fits (black lines). (b) Model populations for the fit to a two-state sequential kinetic model. (c) Evolution-associated spectra (EAS) for the three states in the model 


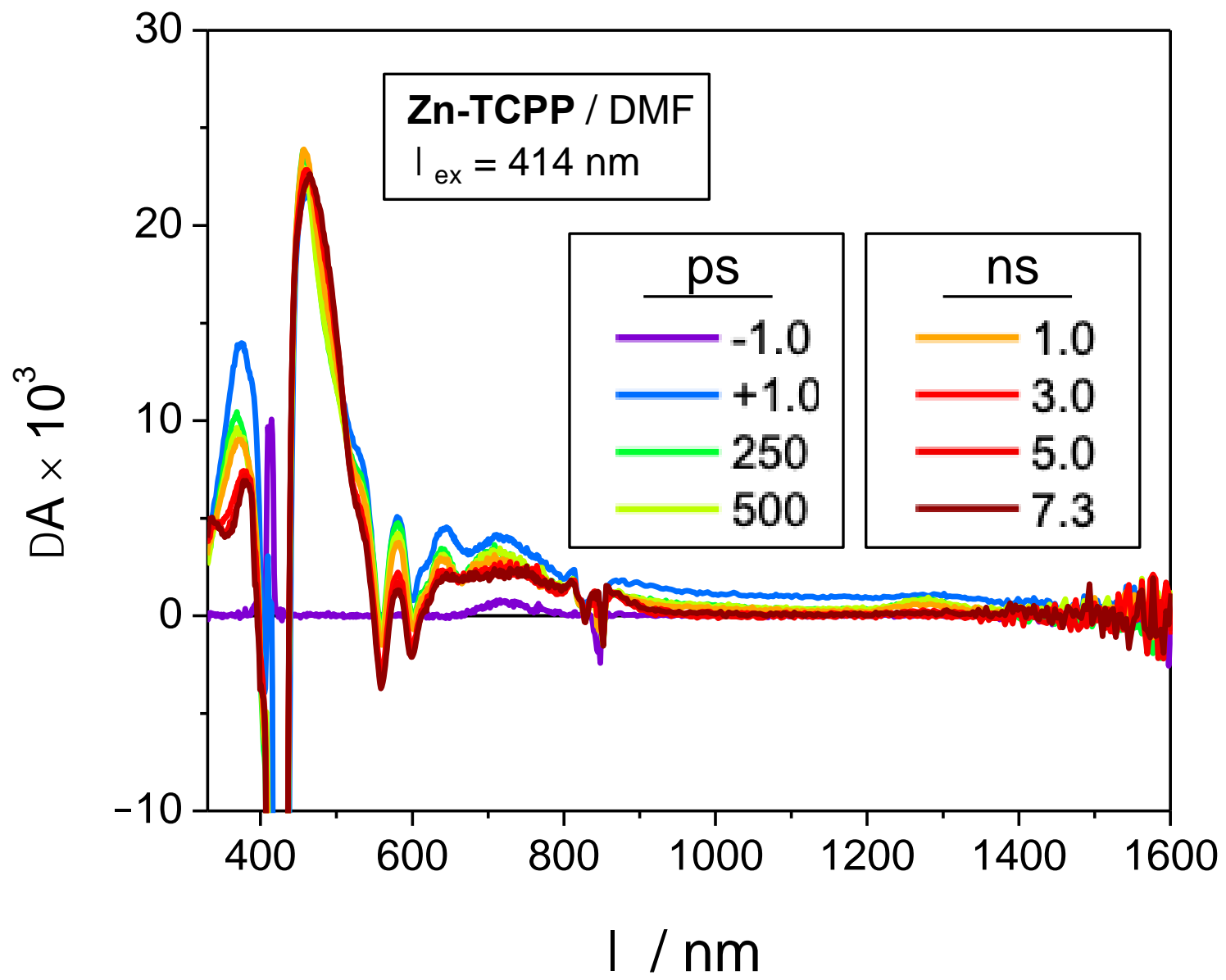

Figure S16. Femtosecond transient absorption spectra of Zn-TCPP in DMF excited at $\lambda_{\mathrm{ex}}=414 \mathrm{~nm}$

Femtosecond TA (Figure S17) shows the second singlet state ( $\left.\mathrm{S}_{2}\right)$ of the porphyrin (A) first of all converts internally in $\sim 1 \mathrm{ps}$ when the Soret band is excited. Then this relaxed singlet $\left(\mathrm{S}_{1}\right)$ state (B) relaxes structurally in $12 \mathrm{ps}$ to state $\mathrm{C}$, which then intersystem crosses in $\sim 1.9 \mathrm{~ns}$ to form the triplet state (D) that lives into the nsTA regime. There is an additional non-resonant solvent signal (state E) that decays within $100 \mathrm{fs}$. This signal is an artifact and not intrinsic to the sample, but was required to be able to fit the data in a proper manner. 


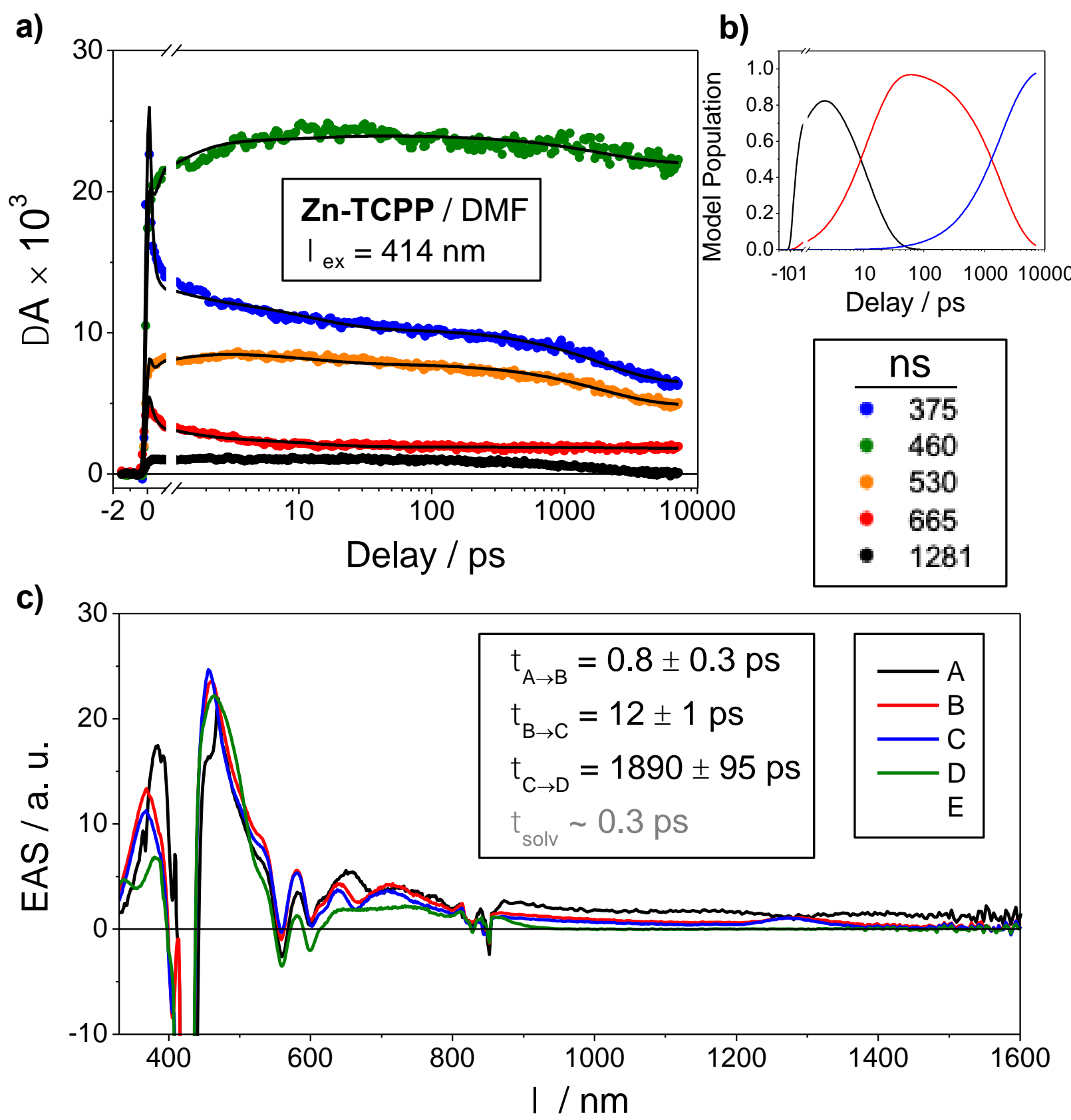

Figure S17. Kinetic analysis of femtosecond transient data for Zn-TCPP in DMF excited at $\lambda_{\mathrm{ex}}=$ $414 \mathrm{~nm}$. (a) Kinetic traces at selected wavelengths with fits (black lines). (b) Model populations for the fit to a five-state sequential kinetic model. (c) Evolution-associated spectra (EAS) for the three states in the model 


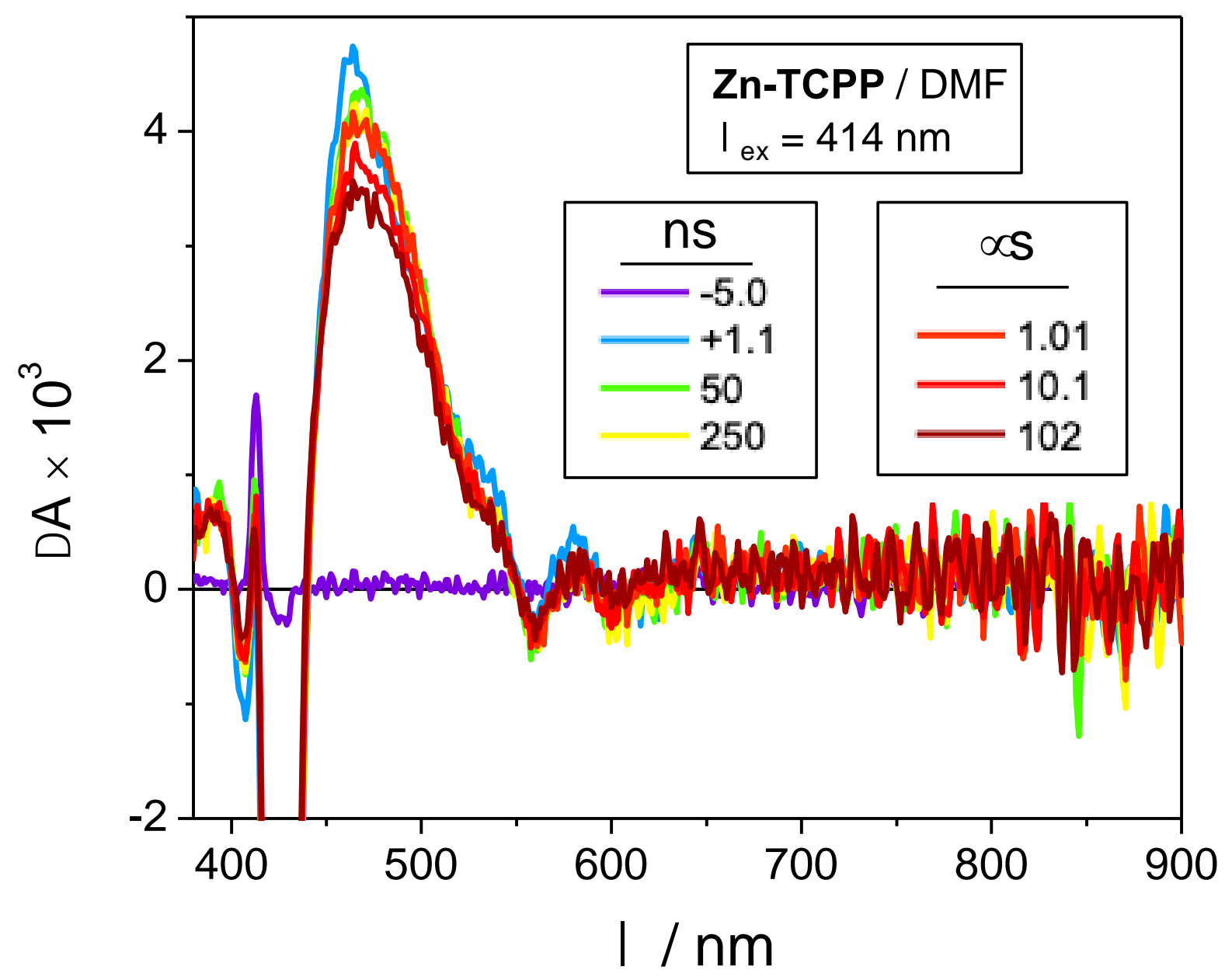

Figure S18. Nanosecond transient absorption spectra of Zn-TCPP in DMF excited at $\lambda_{\mathrm{ex}}=414 \mathrm{~nm}$

Nanosecond TA shows (Figure S19) the singlet state (C) intersystem crosses in 2.2 ns to form the triplet state (D) that shows some relaxation in $\sim 1.2$ microseconds to state $\mathrm{E}$, which then decays to the ground state in $\sim 950$ microseconds. 
a)

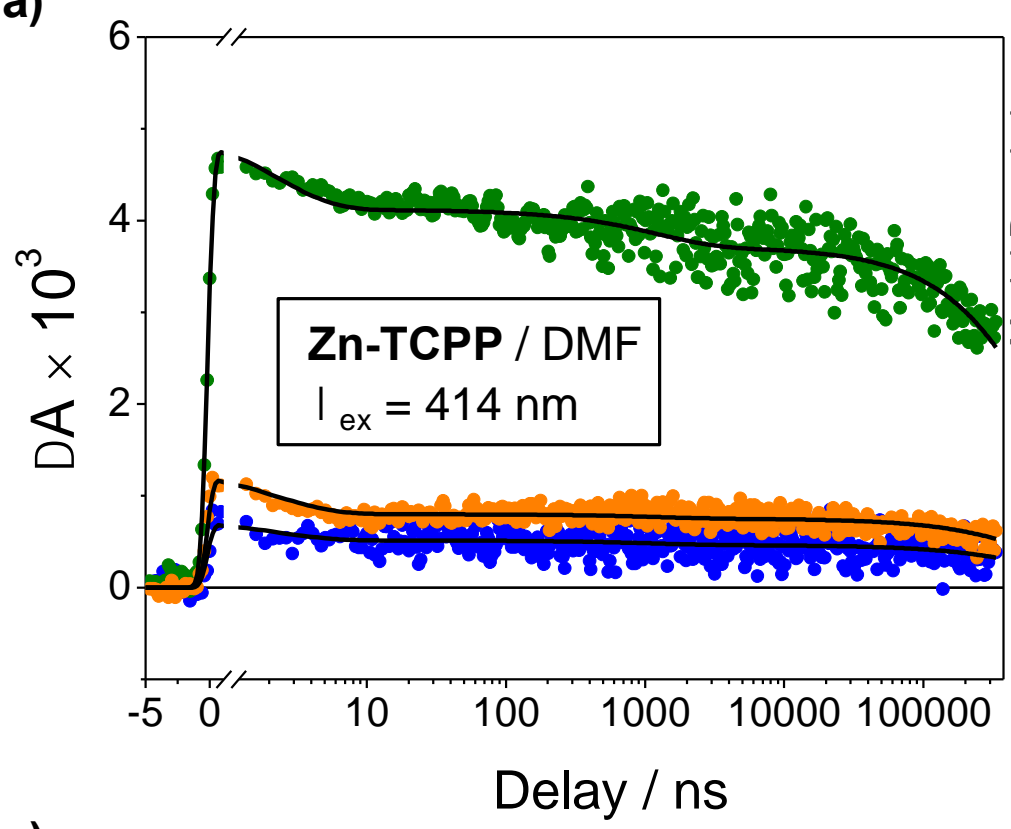

b)
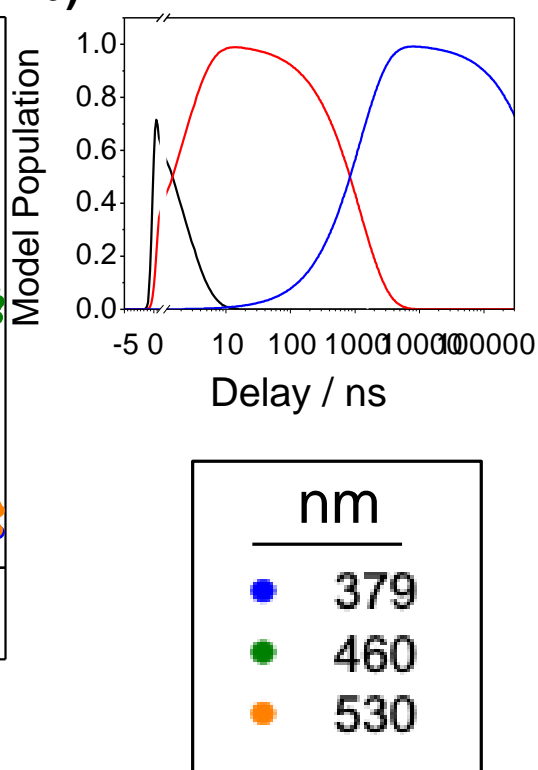

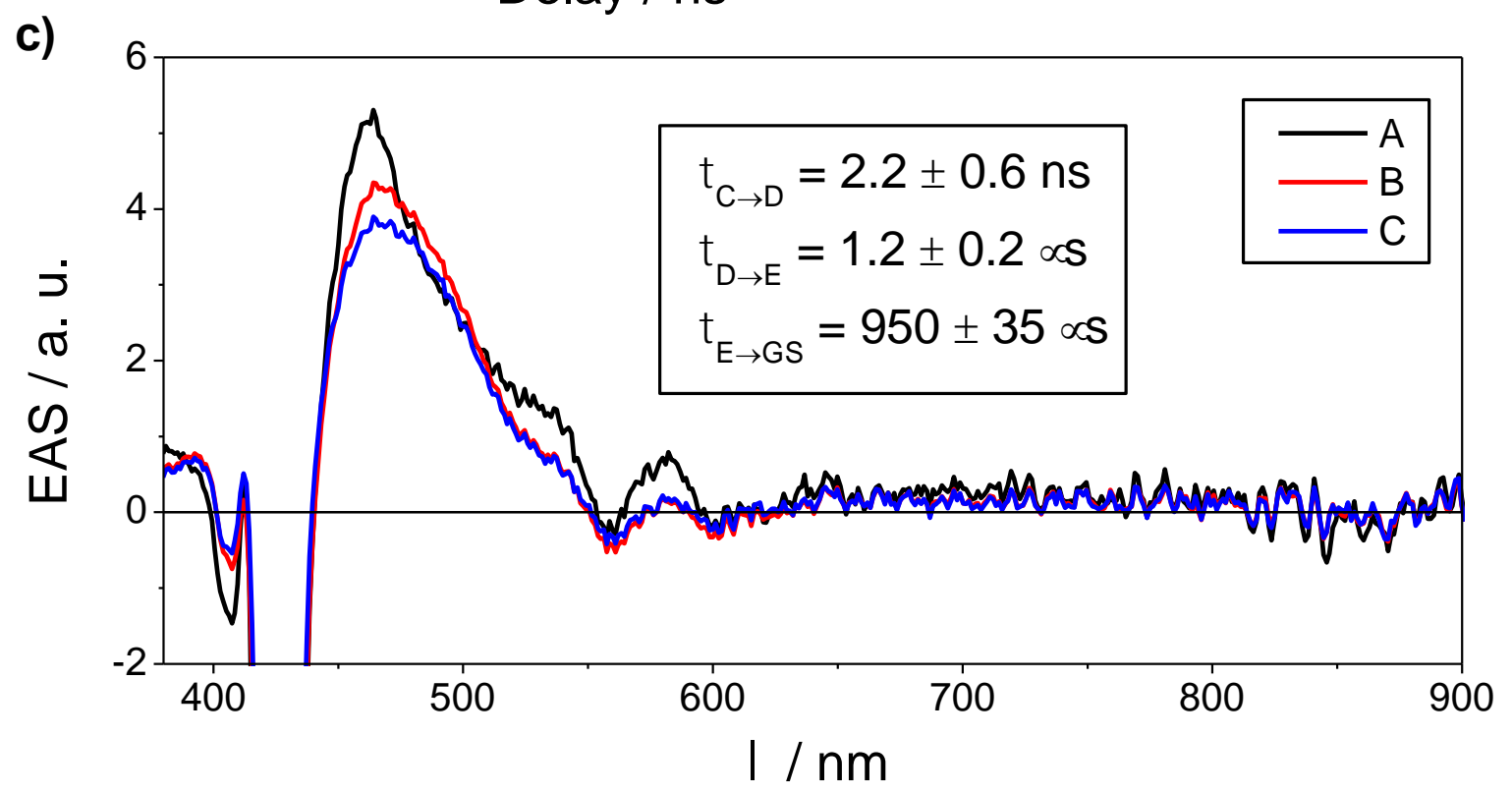

Figure S19. Kinetic analysis of nanosecond transient data for Zn-TCPP in DMF excited at $\lambda_{\mathrm{ex}}=$ $414 \mathrm{~nm}$. (a) Kinetic traces at selected wavelengths with fits (black lines). (b) Model populations for the fit to a three-state sequential kinetic model. (c) Evolution-associated spectra (EAS) for the three states in the model 


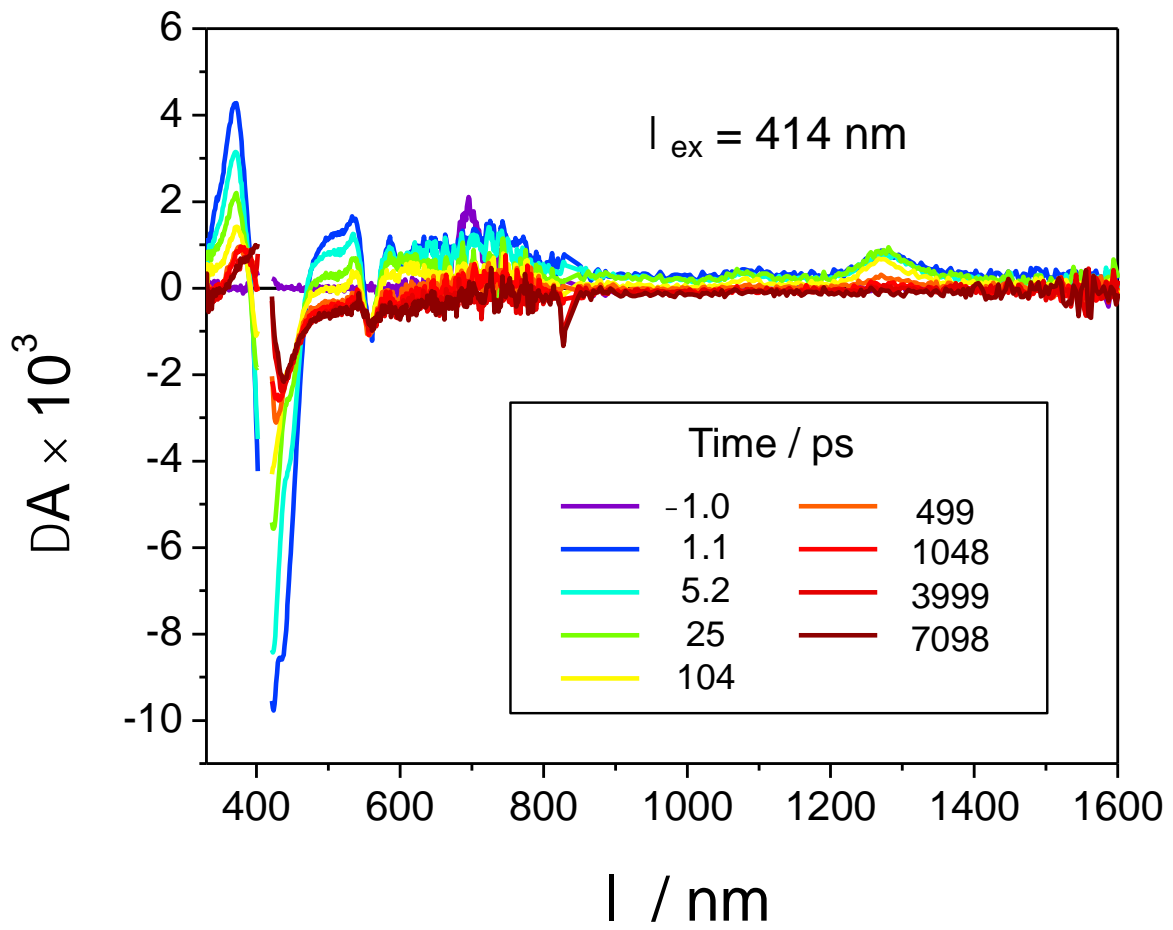

Figure S20. Femtosecond transient absorption spectra of TzPMOF in $\mathrm{MeCN}$ excited at $\lambda_{\mathrm{ex}}=414 \mathrm{~nm}$

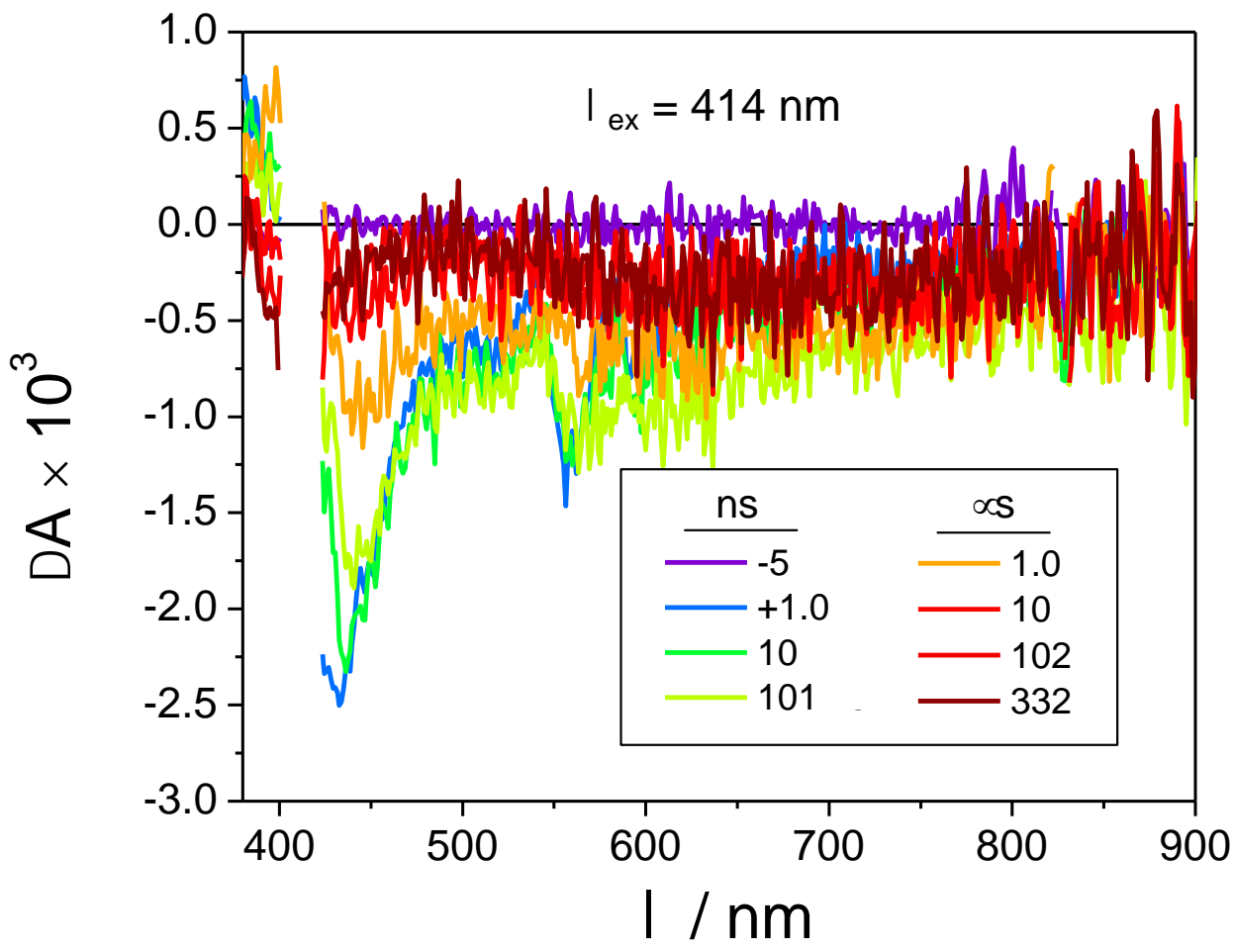

Figure S21. Nanosecond transient absorption spectra of TzPMOF in $\mathrm{MeCN}$ excited at $\lambda_{\mathrm{ex}}=414 \mathrm{~nm}$ 


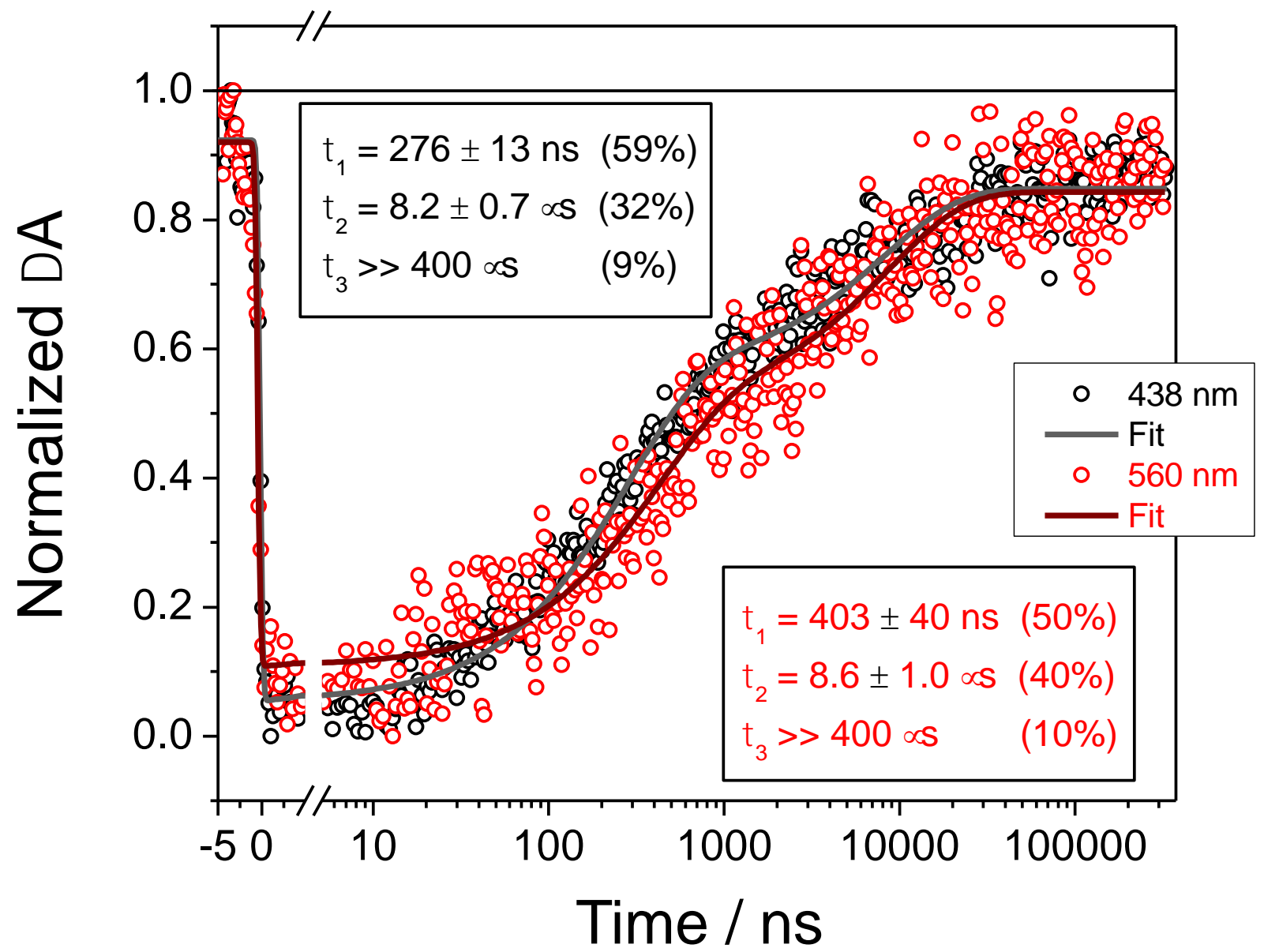

Figure S22. Decay profiles of TzPMOF in MeCN

The nsTA shows that the triplet state lifetime is on the microsecond timescale, which is long enough for triplet exciton hopping to allow multiple triplets to meet and perform the upconversion. 


\section{Section F. Steady-State Microscopy}

a)
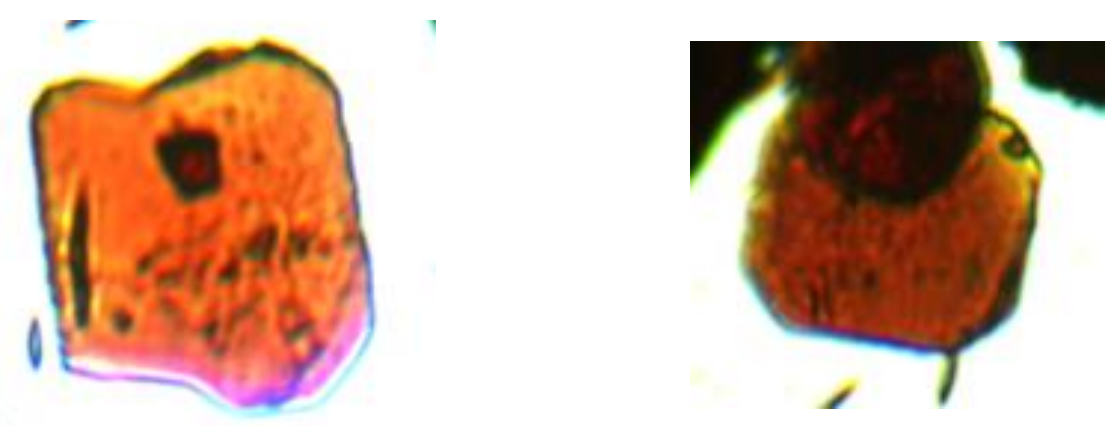

Crystal 1

Crystal 2

b)

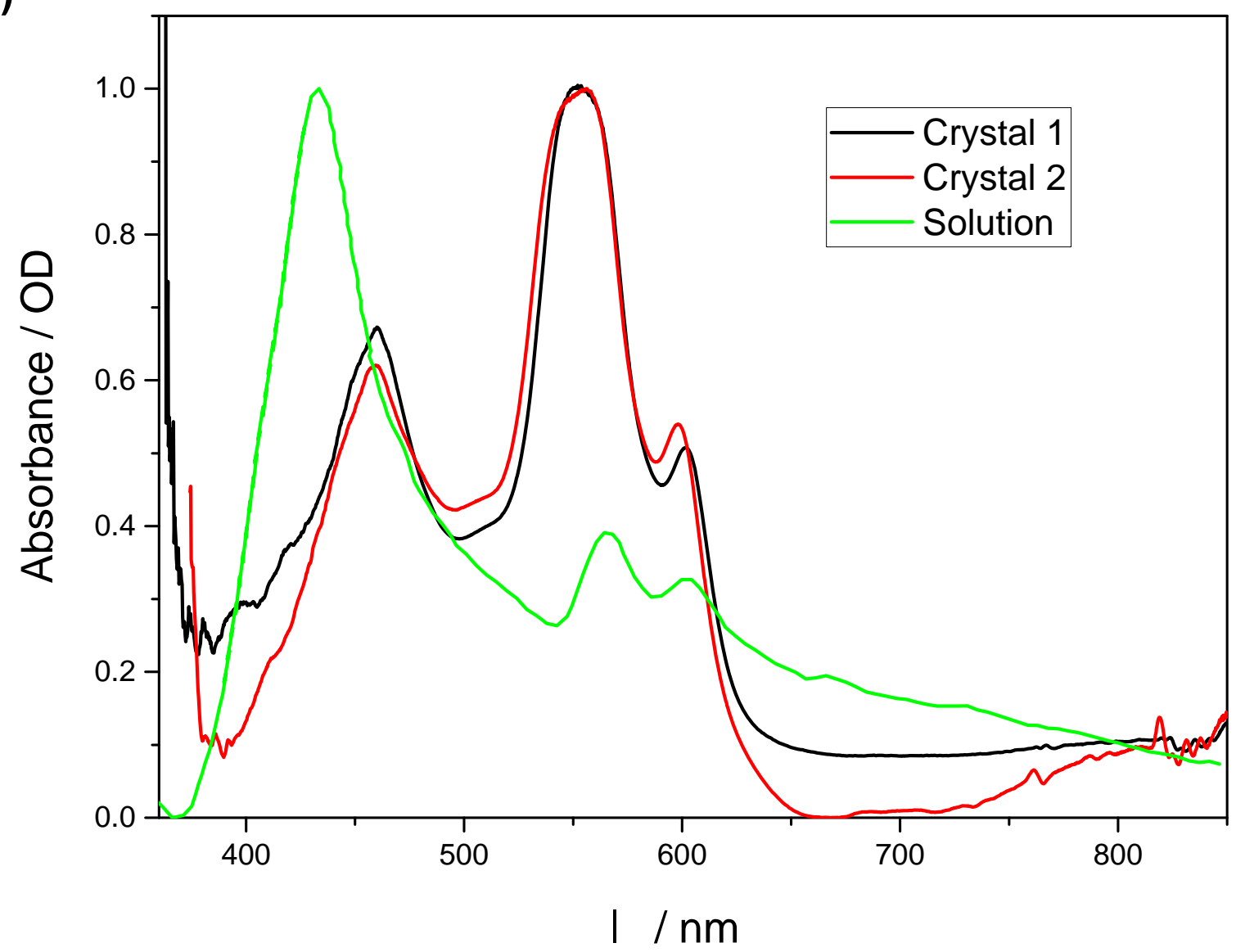

Figure S23. (a) TzPMOF crystals 1 and 2, size : $20 \times 20 \mu \mathrm{M}^{2}$. (b) UV-Vis spectra of TzPMOF single crystals 1 and 2 and TzPMOF suspension in $(\mathrm{Me})_{2} \mathrm{CO}$. 


\section{Section G. Transient Absorption Microscopy}

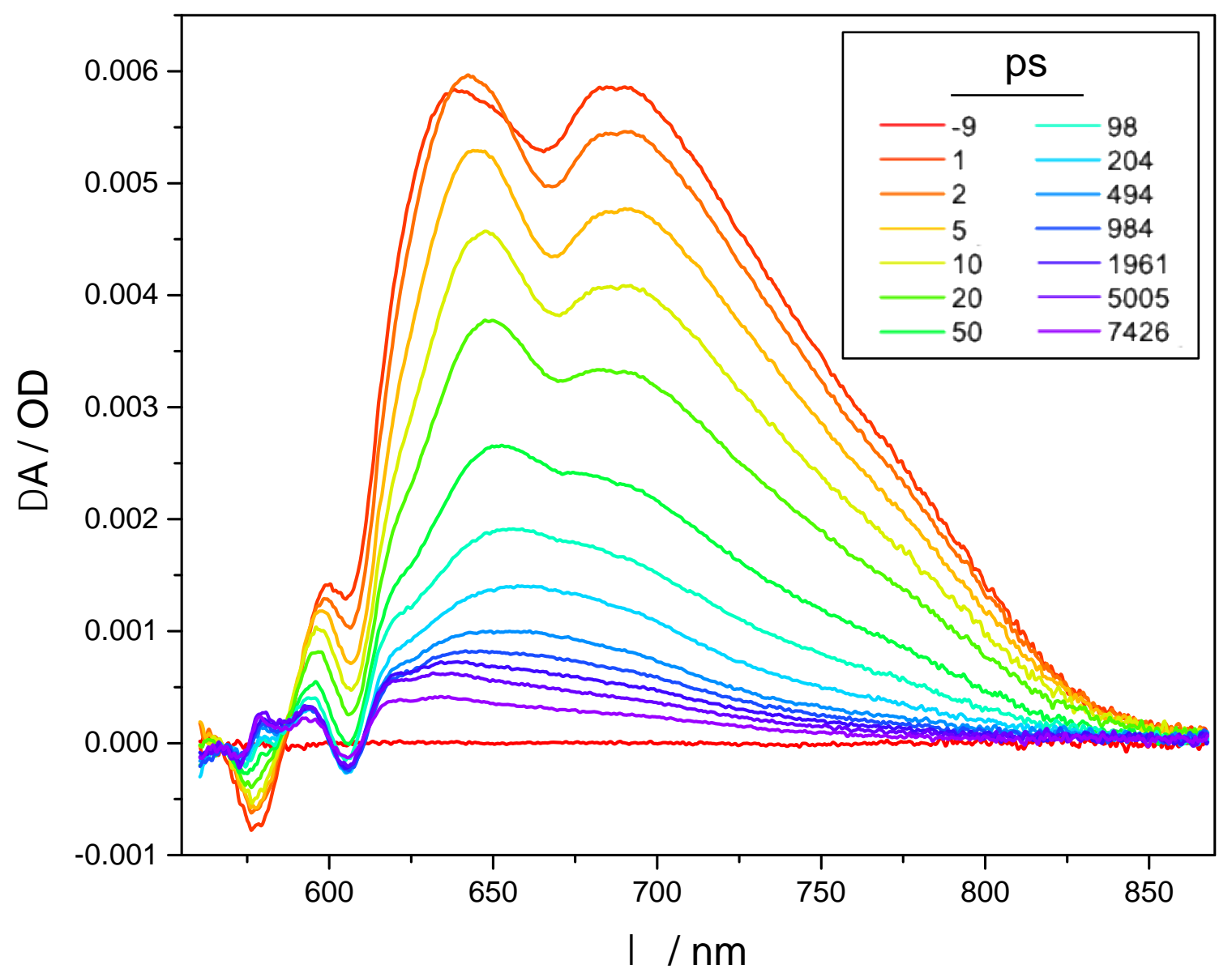

Figure S24. Femtosecond transient absorption spectra of TzPMOF single crystal excited at $\lambda_{\mathrm{ex}}=542 \mathrm{~nm}$ 
a)

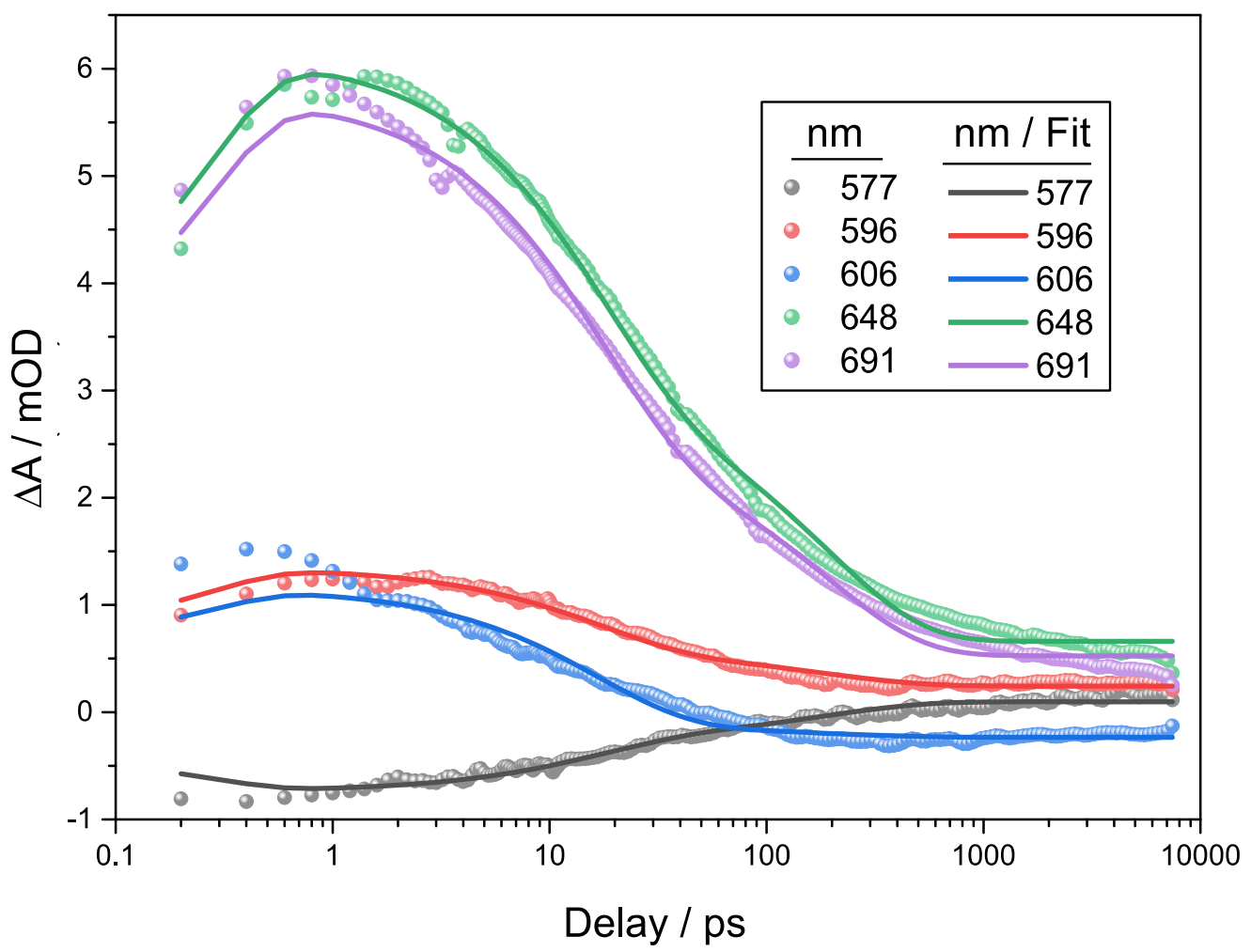

b)

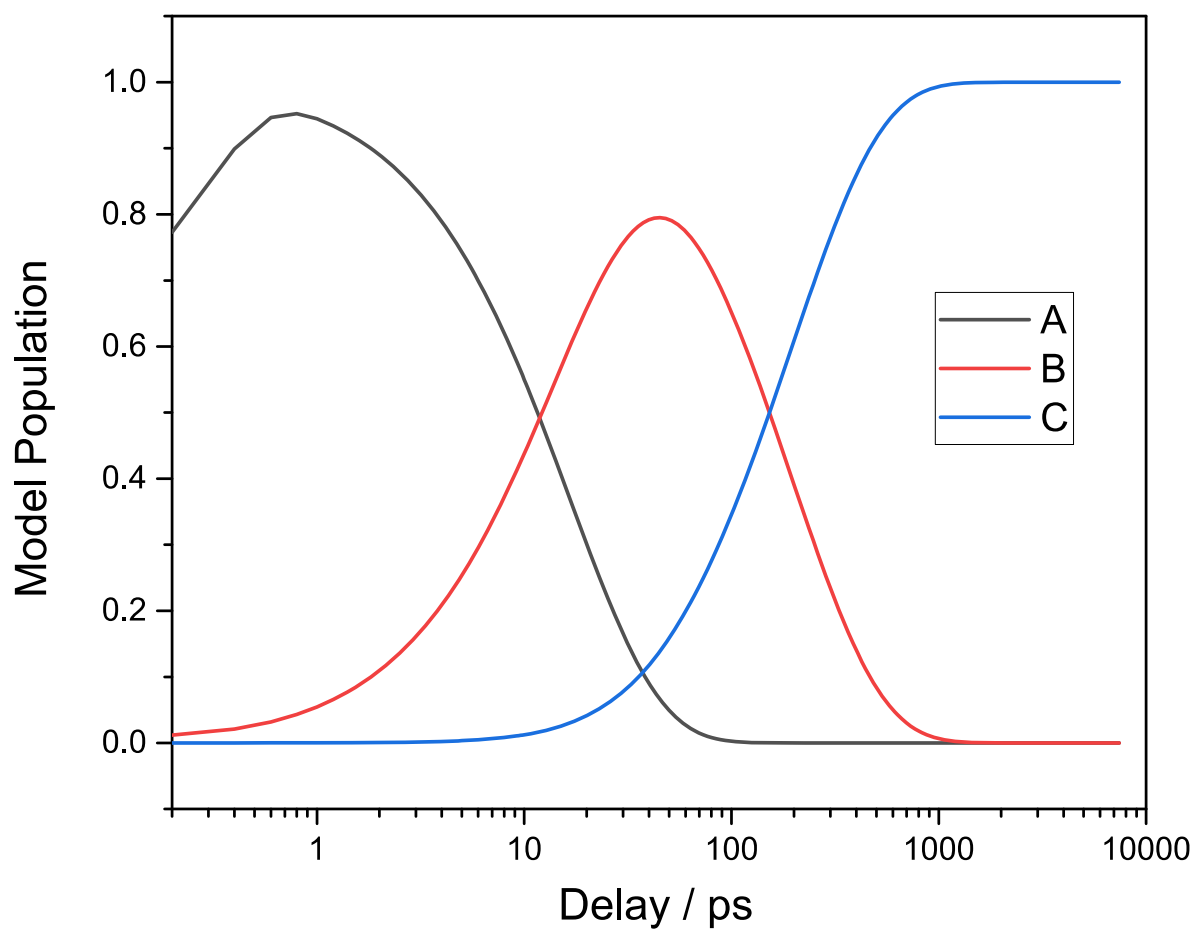

Figure S25. Kinetic analysis of femtosecond transient microscopy data for TzPMOF single crystal in DMF excited at $\lambda_{\mathrm{ex}}=542 \mathrm{~nm}$. (a) Kinetic traces at selected wavelengths with fits. (b) Model populations for the fit to a three-state sequential kinetic model. 


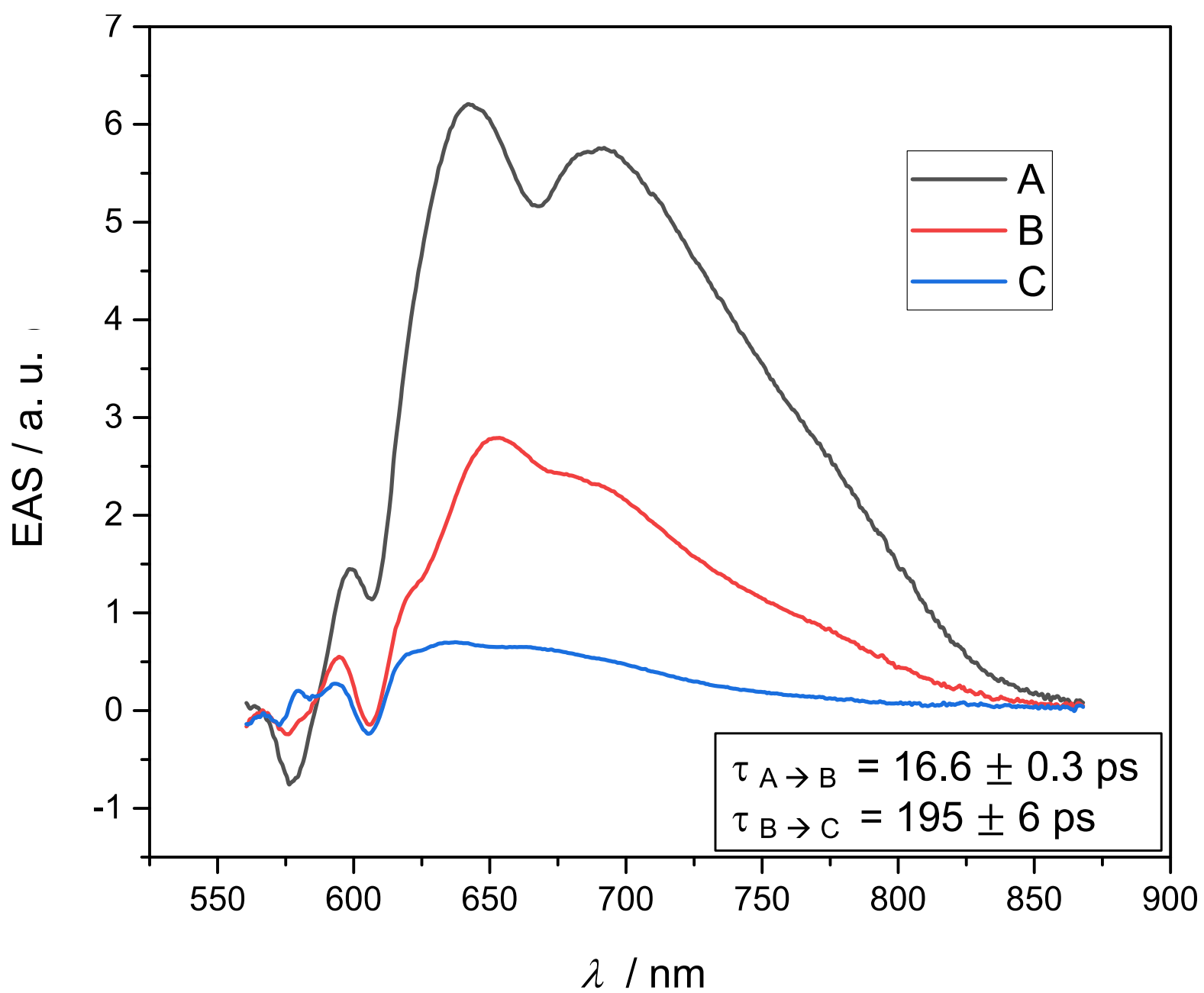

Figure S26. Evolution-associated spectra (EAS) for the three states in the model: state A is the initially excited singlet excited state, state B is the charge-separated state, and state C is the triplet state. 


\section{Section H. References}

1. Young, R. M., Dyar, S. M., Barnes, J. C., Juríček, M., Stoddart, J. F., Co, D. T. \& Wasielewski, M. R. Ultrafast conformational dynamics of electron transfer in ExBox ${ }^{4+} \subset$ Perylene. J. Phys. Chem. A 117, 12438-12448 (2013).

2. The MathWorks, Inc., Natick, Massachusetts, United States.

3. Roy, I., Bobbala, S., Zhou, J., Nguyen, M. T., Nalluri, S. K. M., Wu, Y., Ferris, D. P., Scott, E. A., Wasielewski, M. R. \& Stoddart, J. F. ExTzBox: A glowing cyclophane for live-cell imaging. J. Am. Chem. Soc. 140, 7206-7212 (2018).

4. Sheldrick, G. M. SHELXT-Integrated space-group and crystal-structure determination. Acta Cryst. Sect. A 71, 3-8 (2015).

5. Sheldrick, G. M. A short history of SHELX. Acta Cryst. A 64, 112-122 (2008).

6. Dolomanov, O. V., Bourhis, L. J., Gildea, R. J., Howard, J. A. K. \& Puschmann, H. OLEX2: A complete structure solution, refinement and analysis program. J. Appl. Crystallogr. 42, 339-341 (2009). 